\title{
Actively variable-spectrum optoelectronics with black phosphorus
}

Hyungjin Kim ${ }^{1,2}$, Shiekh Zia Uddin ${ }^{1,2}$, Der-Hsien Lien ${ }^{1,2}$, Matthew Yeh $^{1,2}$, Nima Sefidmooye Azar ${ }^{3}$, Sivacarendran Balendhran ${ }^{4}$, Taehun Kim ${ }^{1,2}$, Niharika Gupta ${ }^{1,2}$, Yoonsoo Rho ${ }^{5}$, Costas P. Grigoropoulos ${ }^{5}$, Kenneth B. Crozier ${ }^{3,4,6} \&$ Ali Javey $^{1,2 *}$

${ }^{1}$ Department of Electrical Engineering and Computer Sciences, University of California, Berkeley, CA, USA.

${ }^{2}$ Materials Sciences Division, Lawrence Berkeley National Laboratory, Berkeley, CA, USA.

${ }^{3}$ Department of Electrical and Electronic Engineering, University of Melbourne, Melbourne, Victoria, Australia.

${ }^{4}$ School of Physics, University of Melbourne, Melbourne, Victoria, Australia.

${ }^{5}$ Department of Mechanical Engineering, University of California, Berkeley, CA, USA.

${ }^{6}$ Australian Research Council (ARC) Centre of Excellence for Transformative Meta-Optical Systems (TMOS), University of Melbourne, Melbourne, Victoria, Australia.

*Corresponding author. E-mail: ajavey@berkeley.edu

Room-temperature optoelectronic devices that operate at shortwave and midwave infrared wavelengths $(1-8 \mu \mathrm{m})$ can be used for numerous applications ${ }^{1-5}$. To achieve the operating wavelength range needed for a given application, a combination of materials with different bandgaps (e.g. superlattice/heterostructure) ${ }^{6,7}$ or the variation of semiconductor alloy composition during growth ${ }^{8,9}$ is used; however, these approaches involve fabrication complexity and the operating range is fixed post-fabrication. Although wide-range, active, and reversible tunability of the operating wavelengths in optoelectronic devices after fabrication is a highly desirable feature, no such platform has been yet developed. Here, we demonstrate high-performance room-temperature infrared optoelectronics with actively variable spectra by presenting black phosphorus (bP) as an ideal candidate. Enabled by the 
extraordinary sensitivity of its bandgap to strain, which varies from 0.22 to $0.53 \mathrm{eV}$, we show the continuous and reversible tuning of the operating wavelengths in bP light-emitting diodes and photodetectors. Furthermore, we leverage this platform to demonstrate multiplexed non-dispersive infrared gas sensing where multiple gases (e.g. $\mathrm{CO}_{2}, \mathrm{CH}_{4}$, and $\mathrm{H}_{2} \mathrm{O}$ ) are detected using a single light source. With its active spectral tunability while retaining high performance, our work bridges a technological gap, presenting a potential route to meet different emission/detection spectrum requirements in various optoelectronic applications. 
The spectral range over which an optoelectronic device can operate is largely dictated by the bandgap of its photoactive material. To achieve the operating range needed for a given application, materials with varying compositions (i.e. by alloying to tune the bandgap) or complex structures consisting of materials with different bandgaps (e.g. superlattices or heterostructure) are used ${ }^{10,11}$. While effective, these come with increased fabrication complexity as they involve the precise control of material thickness and/or composition. In addition, these approaches produce devices whose operating wavelength ranges are fixed post fabrication. Although the absorption edge can be shifted by applying an electric field for Franz-Keldysh effect or Stark effect ${ }^{12,13}$, the decrease in photoresponse and luminescence intensity at the extended spectral range as well as the requirement for additional gate electrodes to apply the bias field is a major bottleneck for developing tunable optoelectronics with high performance.

Strain is an effective knob to modulate the electronic and optical properties of semiconductors ${ }^{14-16}$, which can achieve reversible and controllable tuning of the bandgap in semiconductors. Whereas only a limited amount of strain can be epitaxially applied in bulk crystalline materials, two-dimensional (2D) materials can sustain much larger elastic strain, thus allowing for wide tuning of their optical bandgap ${ }^{17,18}$. Due to its puckered lattice structure, black phosphorus (bP) specifically has shown unique strain-dependent properties, including bandgap which is highly sensitive to $\operatorname{strain}^{19-22}$, anomalous strain dependence of the bandgap ${ }^{23}$, tunable van der Waals interaction ${ }^{24}$, and piezoelectricity ${ }^{25}$. Strain can be applied in a reversible manner in bP due to its membrane nature which does not require a selective thinning and lift-off process ${ }^{26,27}$. The bandgap of multilayer bP also spans the portion of the IR region in which there is a rapidly increasing demand for optical communications, thermal imaging, health monitoring, spectroscopy, 
and gas sensing ${ }^{28}$. Moreover, the low Auger recombination velocity ${ }^{29,30}$ and direct bandgap of bP at all thicknesses are advantageous for high-efficiency optoelectronic applications. However, despite considerable interest in developing actively tunable IR optoelectronic devices, no significant progress has been made in that direction.

In this study, we utilize the strain-tunable bandgap of bP for the demonstration of actively variable spectrum optoelectronic (AVSO) devices that operate in the infrared regime at room temperature. Covering from $2.3 \mu \mathrm{m}$ to $5.5 \mu \mathrm{m}$, the bP bandgap exhibits a modulation rate of 1.70 $\mu \mathrm{m} / \%$ by strain using our methods, which is the highest value reported for direct bandgap semiconductors. This enables wide range modulation of their spectral range in both light-emitting and detecting devices at will without sacrificing their performance. Continuous and reversible tuning of mid-wavelength infrared (MWIR) electroluminescence (EL) is demonstrated using bP$\mathrm{MoS}_{2}$ heterostructure on a flexible substrate. The detection range of highly-responsive bP photodetectors is additionally extended by applied strain. Finally, we detect multiple gases (e.g. $\mathrm{CO}_{2}, \mathrm{CH}_{4}$, and $\mathrm{H}_{2} \mathrm{O}$ ) using a single tunable IR light source. Owing to its reversibility and capacity for active spectral tunability while retaining high performance, our approach provides versatility in various fields where a tunable spectrum is required at room temperature.

Strain-tunable bandgap in bP. BP has a puckered hexagonal structure with two crystal orientations in each layer: armchair (AC, x) and zigzag (ZZ, y) (Fig. 1a). The strain-induced actively variable bandgap of bP enables AVSO devices at room temperature that exhibit wide and reversible operating wavelength tunability (Fig. 1b). Mechanically exfoliated bP was transferred onto a pre-annealed polyethylene terephthalate glycol-modified (PETG) substrate at $95{ }^{\circ} \mathrm{C}$ (Fig. 1c). The rapid thermal quenching of the bP/PETG sample to room temperature resulted in the 
application of compressive strain to bP due to the coefficient of thermal expansion (CTE) mismatch between bP and PETG (Methods). Thereafter, the sample was bent to apply tensile strain. Here, we quantify the strain via its ZZ (y) direction component (Extended Data Fig. 1a,b). Therefore, the strain in bP in this work is determined by the sum of the compressive (negative) strain in ZZ (y) direction induced by thermal expansion mismatch and tensile (positive) strain in ZZ (y) direction from bending stress. The strain-induced bandgap shift in bP was verified by IR photoluminescence (PL) spectroscopy (Fig. 1d). Before bending, the PL peaked at a wavelength of $5.5 \mu \mathrm{m}(0.66 \%$, compressive). A large spectral blueshift of $3.2 \mu \mathrm{m}$ was observed after mechanical bending $(1.21 \%$, tensile), corresponding to a bandgap shift from $0.22 \mathrm{eV}$ to $0.53 \mathrm{eV}$ by applied strain. At $0 \%$ strain, the PL had a peak position at $0.35 \mathrm{eV}$, which is in good agreement with the PL spectrum of unstrained bP from the literature ${ }^{31}$. Raman studies showed that the shifting rates with the applied strain were consistent with previous reports ${ }^{25,32}$ (Extended Data Fig. 1c-e). In addition, we found that built-in compressive strain plays a major role in preventing any partial relaxation or slippage during bending, retaining up to $1.21 \%$ of tensile strain (Extended Data Fig. 2a). Owing to the effective transfer of mechanical strain and extraordinary sensitivity of its bandgap to strain, our method offers a significantly broad and continuous bandgap tuning range at room temperature. The wavelength range spanned by our approach is from the short-wavelength infrared (SWIR) to the MWIR and could only be accessed otherwise by multiple devices comprising bulk semiconductor alloys with different compositions.

Detailed optical characterization was performed using IRPL spectroscopy to further investigate bandgap tunability in bP using strain. As the strain increased, the PL intensity increased and the wavelength of the spectral peak varied continuously from $5.5 \mu \mathrm{m}$ to $2.3 \mu \mathrm{m}$ (Fig. 2a). The 
blueshift rate of the bP bandgap by strain was found to be $1.70 \mu \mathrm{m} / \%(162 \mathrm{meV} / \%)$, which is substantially higher than other semiconductors with similar bandgaps (e.g. Ge and InAs) (Fig. 2b and Extended Data Table 1). Changing the direction of tensile strain with respect to the crystal orientation of $\mathrm{bP}(\mathrm{AC}$ and $\mathrm{ZZ})$ did not have a large effect on the magnitude of the bandgap shift (Extended Data Fig. 2c), which agrees with previous work ${ }^{22}$. Additionally, light absorption at the PL excitation wavelength showed little dependence on strain (Extended Data Fig. 2d). The relative PL quantum yield (PL QY) in bP under different strains is thus given by the integrated luminous intensity divided by the incident power as well as light absorption (Fig. 2c). Strained bP exhibited a continuous PL QY increase in the high pump regime whereas in unstrained bP the QY remained constant. Because our incident power range is less than two orders of magnitude, which is limited by the measurement setup, it is not sufficient to extract recombination coefficients by fitting the incidence power-dependent data we have. This introduces uncertainty in determining which of parameters (Shockley-Read-Hall (SRH), radiative, or Auger) are affected by strain, with respect to carrier densities. The PL QY in bP under tensile strain (1.21\%) is higher than that in bP under compressive strain $(0.66 \%)$ and unstrained bP. These results can be attributed to the change in effective mass by strain. Namely, the uniaxial tensile strain strongly alters the effective mass, with a blueshift of the bandgap and an increase in the radiative recombination rate. As high current density operation is required in light-emitting devices, this property makes bP under tensile strain a suitable material for high-efficiency infrared light emitting devices.

Strain-tunable MWIR light emission. Recently, an electrically-pumped emission from bP shows great promises as a light source at MWIR wavelengths ${ }^{33-35}$, due to its direct bandgap even in bulk limit and its low Auger recombination velocity ${ }^{29,30}$. By leveraging the property of bP whose 
bandgap can be widely modulated by strain, we developed strain-tunable light-emitting diodes (LEDs) based on bP/MoS 2 heterojunctions (Fig. 3a,b), where tensile strain is applied via bending along the ZZ (y) orientation of bP (Fig. 3c). Here, $\mathrm{MoS}_{2}$ was used as an electron selective contact. Polyimide is used as the substrate to fabricate LEDs because PETG is not able to withstand conventional lithography processes (Methods). As strain was varied from compressive to tensile, the peak in EL spectrum continuously shifted from $4.07 \mu \mathrm{m}(0.20 \%$, compressive) to $2.70 \mu \mathrm{m}$ (1.06 \%, tensile) (Fig. 3d). Similar to the trend observed in our PL results, the EL intensity also increased with tensile strain as the emission wavelength became shorter. The EL peak wavelength as a function of the current density was characterized for different current densities (Extended Data Fig. 3a). The angular intensity distributions of the bP-LED were also simulated at two emission wavelengths $(4.07 \mu \mathrm{m}$ and $2.70 \mu \mathrm{m})$, which were found to be similar (Extended Data Fig. 3b,c). We observed that, at all current densities, the bP-LED under tensile strain showed higher EL intensity than when in compressive strain (Fig. 3e). The EL enhancement with tensile strain was due to the resultant PL QY enhancement, as the injection efficiency did not improve with strain (Extended Data Fig. 3d). The light emission from bP-LED was highly polarized, which is consistent with previous reports ${ }^{33-35}$ (Fig. 3e, inset). Our bP-LED also shows stable emission wavelength tunability above room temperature $\left(20^{\circ} \mathrm{C}<T<100{ }^{\circ} \mathrm{C}\right)$ (Extended Data Fig. 4). However, the emission intensity decreases with increasing temperature, which can be attributed to the thermal activation of the nonradiative recombination centers at high temperature ${ }^{31}$. We extracted the internal quantum efficiency (IQE) of our bP-LED, which is plotted along with the theoretical PL QY of InAs, PbSe, GaSb, and bP as a function of carrier concentration (Fig. 3f). Our device showed a peak IQE of $0.73 \%(0.2 \%$, compressive $)$ and $2.47 \%(1.0 \%$, tensile $)$ at 
room temperature. A detailed discussion of measuring output power to calculate the IQE of the bPLED is provided in Methods. For the case of a semiconductor with low background doping (i.e. $n$ $=p$ ), the theoretical PL QY is calculated according to a standard $A B C$ recombination model as:

$$
\mathrm{QY}=\frac{B\left(n^{2}-n_{i}^{2}\right)}{\frac{A\left(n^{2}-n_{i}^{2}\right)}{n}+B\left(n^{2}-n_{i}^{2}\right)+2 C n\left(n^{2}-n_{i}^{2}\right)}
$$

where $\underline{n}_{\mathrm{i}}$ is the intrinsic carrier concentration and $A, B$, and $C$ are the SRH, radiative, and Auger recombination coefficient, respectively. The parameters for InAs, $\mathrm{PbSe}, \mathrm{GaSb}$, and bP used to calculate QY are summarized in Extended Data Table 2. Since the SRH recombination coefficient is an extrinsic parameter and highly dependent on material quality, it was assumed to be zero for this analysis. For small bandgap semiconductors, the Auger recombination is likely to be pronounced and a limiting factor for performance of LED. This originates from the relationship between the ratio of the Auger lifetime $\left(\tau_{a}\right)$ to the radiative lifetime $\left(\tau_{r}\right)$ and the bandgap $\left(E_{g}\right)$ which is given by:

$$
\frac{\tau_{a}}{\tau_{r}} \propto \exp \left[\left(\frac{\frac{m_{e}^{*}}{m_{h}^{*}}}{1+\frac{m_{e}^{*}}{m_{h}^{*}}}\right)\left(\frac{E_{g}}{k_{B} T}\right)\right]
$$

where $m_{e}^{*}$ and $m_{h}^{*}$ are the effective masses of electrons and holes, respectively, $k_{B}$ is Boltzmann's constant, and $T$ is temperature ${ }^{36,37}$. Since $m_{e}^{*}$ and $m_{h}^{*}$ in bP have similar values, the effective mass ratio $\left(m_{e}^{*} / m_{h}^{*}\right)$ is much higher than that of other small bandgap semiconductors. According to equation (2), this results in suppressed Auger recombination (longer Auger lifetime), which leads to bP's theoretical QY limit being much higher than that of other small bandgap semiconductors in the high injection regime. 
We characterized the cyclic repeatability and stability of emission tuning in our bP-LED, exhibiting reversible and stable emission tuning over 500 bending cycles during $\sim 6$ hours of operation (Fig. 3g,h and Supplementary Video 1). A light source capable of active and continuous tuning of its operating wavelength within this spectral region is of particular significance, as this wavelength range consists of various wavelength bands essential for gas detection and chemical analysis. Our strain-tunable bP-LED not only provides wide tuning capability that is equivalent to the integration of numerous devices made from bP with a thickness range of 7-200 nm, but also enhances the performance of MWIR light-emitting devices by applied strains. Furthermore, the low operating power of our bP-LEDs and their potential for monolithic integration with silicon technology ${ }^{35}$ would be advantageous for numerous applications.

NDIR gas-sensing with strain-tunable MWIR LEDs. Non-dispersive infrared (NDIR) gas sensing has attracted great interest due to its fast response and stable lifetime ${ }^{38}$. Thermal emitters (e.g. tungsten filament lamps) are often used in NDIR gas sensors as light sources, but their slow modulation and high power consumption remain key challenges that make them disadvantageous for use in real-time gas monitoring devices with extended lifetime towards the 'Internet of Things ${ }^{39,40}$. Therefore, strain-tunable bP-LEDs with low operating power, fast modulation, and rapid stabilization suggest a solution, permitting multiplexed gas detection with a single light source, where the emission wavelength can be tuned over a broad range to suit various target gases. Our NDIR gas sensing setup (Fig. 4a) and measurement process are detailed in Methods. The strain applied to our bP-LED is varied so that the peak wavelength of the EL spectrum is shifted to match the dominant absorption peaks of $\mathrm{H}_{2} \mathrm{O}, \mathrm{CH}_{4}$, and $\mathrm{CO}_{2}$ (Extended Data Fig. 5a). Since the emission spectrum of our bP-LED under compressive strain had a large overlap with the absorption 
spectrum of $\mathrm{CO}_{2}$ (Fig. 4b), the EL intensity from a bP-LED measured by the detector at $4.3 \mu \mathrm{m}$ was attenuated by the $\mathrm{CO}_{2}$ absorption, enabling detection over a wide range of $\mathrm{CO}_{2}$ concentrations varying from 0.005 to $100 \%$ (Fig. 4d). On the other hand, under $0.3 \%$ tensile strain, the $\mathrm{CH}_{4}$ gas with a concentration range of 0.05 to $2.5 \%$ (50\% of the lower explosive limit) was detected, resulting from the attenuation of measured EL intensity corresponding to $\mathrm{CH}_{4}$ absorption at 3.3 $\mu \mathrm{m}$ (Fig. $4 \mathrm{c}, \mathrm{e}$ ). Selectivity of gas sensing between $\mathrm{CO}_{2}$ and $\mathrm{CH}_{4}$ and the long-term stability of the setup were also characterized (Extended Data Fig. 5b-e). The EL intensity measured by the photodiode as a function of target gas concentrations was fit using the modified Beer-Lambert law model, given by FA $=\left(I_{N_{2}}-I_{x}\right) / I_{N_{2}}=1-\exp (-k L x)$ where FA is fractional absorbance of the target gas, $I_{N_{2}}$ and $I_{x}$ are the EL intensity measured by the photodiode under a nitrogen purge and at a target gas concentration of $x$, respectively, $k$ is a fitting constant, and $L$ is the chamber length (2") (Fig. $4 \mathrm{~d}, \mathrm{e}$, inset). By leveraging the repeatable and continuous tuning capability of our strain-tunable bP-LED, we demonstrated multiplexed gas sensing (Fig. 4f). Responses from the individual gases $\left(\mathrm{CH}_{4}\right.$ only, $\mathrm{CO}_{2}$ only, and water only) are measured to characterize the selectivity of our multiplexed gas sensing system. A sequence of responses from a mixture gas pulse consisting of $0.2 \% \mathrm{CO}_{2}, 2 \% \mathrm{CH}_{4}$, and $\mathrm{H}_{2} \mathrm{O}$ at $20 \%$ relative humidity (R.H.) indicates that $\mathrm{H}_{2} \mathrm{O}(1.0 \%$ tensile strain), $\mathrm{CH}_{4}$ (0.3\% tensile strain), and $\mathrm{CO}_{2}$ (compressive strain) are detected with a single light source, upon the application of different strains to tune the peak emission wavelength of the bPLED.

Strain-tunable photodetection. Photodetectors based on bP have been extensively studied ${ }^{41-48}$. In this work, the same concept was applied to realize actively tunable photodetection with bP. Spectrally tunable bP-based photoconductors are fabricated on a PETG substrate to apply strain 
(Extended Data Fig. 6a,b). As the tensile strain was applied in the ZZ (y) orientation of bP (Extended Data Fig. 6c), the cutoff wavelength (the wavelength at which the responsivity is $50 \%$ of the peak value) of the bP photoconductor was continuously tuned from $4.32 \mu \mathrm{m}(0.4 \%$, compressive) to $2.44 \mu \mathrm{m}(1.0 \%$, tensile) (Extended Data Fig. 6d). Our strain-tunable bP photoconductor shows a peak $D^{*}$ of $5.97 \times 10^{9} \mathrm{~cm} \mathrm{~Hz}^{1 / 2} \mathrm{~W}^{-1}$ at $\lambda=4.0 \mu \mathrm{m}(0.4 \%$, compressive $)$ and a peak $D^{*}$ of $8.45 \times 10^{9} \mathrm{~cm} \mathrm{~Hz}^{1 / 2} \mathrm{~W}^{-1}$ at $\lambda=2.0 \mu \mathrm{m}(1.0 \%$, tensile) (Extended Data Fig. $7 \mathrm{c}$ ), which are promising among the commercially available photodetectors, especially for room temperature operations. This suggests that our strategy can bridge the photodetection gap in the wavelength range between SWIR and MWIR. The strain-tunable bP photoconductor thus combines both benefits, i.e. high detectivity at room temperature and broadband tuning capability, arising from the highly strain-sensitive $\mathrm{bP}$ bandgap ${ }^{19-22}$. Detailed characterization and analysis for photodetection are provided in Methods and Extended Data Fig. 6 and 7.

\section{Conclusion}

In summary, we utilized the strain-tunable bandgap of bP to develop actively tunable IR optoelectronic devices including LEDs and photodetectors at room temperature. The AVSO devices developed here have unprecedented versatility and thus important implications in fields such as optical communications, chemical sensing, and spectroscopy, where a tunable spectrum is required. To this end, we demonstrated one such application of this tunable optoelectronic platform by performing multiplexed gas sensing with a single device. By expanding upon this approach, one could potentially explore integration of devices on piezoelectric substrates or utilizing MEMS structure although there are a number of processing challenges that need to be overcome. Furthermore, it may be possible to extend to long-wave infrared (LWIR) wavelengths by further 
increasing the amount of compressive strain applied to bP through substrate engineering. This active strain tuning scheme may be applicable to other $2 \mathrm{D}$ materials as well, providing a path towards spectrally tunable optoelectronics across the electromagnetic spectrum. 


\section{References}

1. Kahn, J. M. \& Barry, J. R. Wireless infrared communications. Proc. IEEE 85, 265-298 (1997).

2. Vollmer, M. \& Mollmann, K.-P. Infrared thermal imaging: fundamentals, research and applications. (Wiley-VCH, Weinheim, ed. 2, 2018).

3. Bagavathiappan, S., Lahiri, B. B., Saravanan, T., Philip, J. \& Jayakumar, T. Infrared thermography for condition monitoring - a review. Infrared Phys. Technol. 60, 35-55 (2013).

4. Baker, M. J. et al. Using Fourier transform IR spectroscopy to analyze biological materials. Nat. Protoc. 9, 1771-1791 (2014).

5. Gibson, D. \& Macgregor, C. A novel solid state non-dispersive infrared $\mathrm{CO}_{2}$ gas sensor compatible with wireless and portable deployment. Sensors 13, 7079-7103 (2013).

6. Haugan, H. J., Szmulowicz, F., Brown, G. J. \& Mahalingam, K. Bandgap tuning of InAs/GaSb type-II superlattices for mid-infrared detection. J. Appl. Phys. 96, 2580-2585 (2004).

7. Kang, J., Tongay, S., Zhou, J., Li, J. \& Wu, J. Band offsets and heterostructures of twodimensional semiconductors. Appl. Phys. Lett. 102, 012111 (2013).

8. Wu, J. et al. Universal bandgap bowing in group-III nitride alloys. Solid State Commun. 127, 411-414 (2003). 
9. Ning, C.-Z., Dou, L. \& Yang, P. Bandgap engineering in semiconductor alloy nanomaterials with widely tunable compositions. Nat. Rev. Mater. 2, 17070 (2017).

10. Yang, Z. et al. Single-nanowire spectrometers. Science 365, 1017-1020 (2019).

11. Kramer, I. J., Levina, L., Debnath, R., Zhitomirsky, D., \& Sargent, E. H. Solar cells using quantum funnels. Nano Lett. 11, 3701-3706 (2011).

12. Whitney, W. S. et al. Field effect optoelectronic modulation of quantum-confined carriers in black phosphorus. Nano Lett. 17, 78-84 (2017).

13. Liu, Y. et al. Gate-tunable giant stark effect in few-layer black phosphorus. Nano Lett. 17, 1970-1977 (2017).

14. Yablonovitch, E. \& Kane, E. O. Band structure engineering of semiconductor lasers for optical communications. J. Lightwave Technol. 6, 1292-1299 (1988).

15. Thompson, S. E. et al. A 90-nm logic technology featuring strained-silicon. IEEE Trans. Electron Dev. 51, 1790-1797 (2004).

16. Chen, Y. et al. Strain engineering and epitaxial stabilization of halide perovskites. Nature 577, 209 (2020).

17. Lee, C., Wei, X., Kysar, J. W. \& Hone, J. Measurement of the elastic properties and intrinsic strength of monolayer graphene. Science 321, 385-388 (2008).

18. Bertolazzi, S., Brivio, J. \& Kis, A. Stretching and breaking of ultrathin $\mathrm{MoS}_{2}$. ACS Nano 5, 9703-9709 (2011). 
19. Rodin, A. S., Carvalho, A. \& Neto, A. C. Strain-induced gap modification in black phosphorus. Phys. Rev. Lett. 112, 176801 (2014).

20. Quereda, J. et al. Strong modulation of optical properties in black phosphorus through strain-engineered rippling. Nano Lett. 16, 2931-2937 (2016).

21. Zhang, Z. et al. Strain-modulated bandgap and piezo-resistive effect in black phosphorus field-effect transistors. Nano Lett. 17, 6097-6103 (2017).

22. Zhang, G. et al. Infrared fingerprints of few-layer black phosphorus. Nat. Commun. 8, 14071 (2017).

23. Çakır, D., Sahin, H. \& Peeters, F. M. Tuning of the electronic and optical properties of single-layer black phosphorus by strain. Phys. Rev. B 90, 205421 (2014).

24. Huang, S. et al. Strain-tunable van der Waals interactions in few-layer black phosphorus. Nat. Commun. 10, 2447 (2019).

25. Ma, W. et al. Piezoelectricity in multilayer black phosphorus for piezotronics and nanogenerators. Adv. Mat. 32, 1905795 (2020).

26. Sanchez-Perez, J. R. et al. Direct-bandgap light-emitting germanium in tensilely strained nanomembranes. Proc. Natl Acad. Sci. USA 108, 18893-18898 (2011).

27. Takei, K. et al. Quantum confinement effects in nanoscale-thickness InAs membranes. Nano Lett. 11, 5008-5012 (2011).

28. Ling, X., Wang, H., Huang, S., Xia, F. \& Dresselhaus, M. S. The renaissance of black phosphorus. Proc. Natl Acad. Sci. USA 112, 4523-4530 (2015). 
29. Ge, S. et al. Dynamical evolution of anisotropic response in black phosphorus under ultrafast photoexcitation. Nano Lett. 15, 4650-4656 (2015).

30. Bhaskar, P., Achtstein, A.W., Vermeulen, M. J. W. \& Siebbeles, L. D. A. Radiatively dominated charge carrier recombination in black phosphorus. J. Phys. Chem. C 120, 13836-13842 (2016).

31. Chen, C. et al. Bright mid-infrared photoluminescence from thin-film black phosphorus. Nano Lett. 19, 1488-1493 (2019).

32. Du, Y. et al. Auxetic black phosphorus: a 2D material with negative Poisson's ratio. Nano Lett. 16, 6701-6708 (2016).

33. Wang, J. et al. Mid-infrared polarized emission from black phosphorus light-emitting diodes. Nano Lett. 20, 3651-3655 (2020).

34. Zong, X. et al. Black phosphorus-based van der Waals heterostructures for mid-infrared light-emission applications. Light: Sci. Appl. 9, 114 (2020).

35. Chang, T.-Y. et al. Black phosphorus mid-infrared light-emitting diodes integrated with silicon photonic waveguides. Nano Lett. 20, 6824-6830 (2020).

36. Haug, A. Auger recombination in direct-gap semiconductors: band-structure effects. $J$. Phys. C: Solid State Phys. 16, 4159 (1983).

37. Kurtz, S. R., Biefeld, R. M. \& Dawson, L. R. Modification of valence-band symmetry and Auger threshold energy in biaxially compressed InAs 1-x $_{\text {Sb }}$. Phys. Rev. B 51, 7310 (1995).

38. Lee, D. D. \& Lee, D. S. Environmental gas sensors. IEEE Sens. J. 1, 214-224 (2001). 
39. Dinh, T.-V., Choi, I.-Y., Son, Y.-S. \& Kim, J.-C. A review on non-dispersive infrared gas sensors: improvement of sensor detection limit and interference correction. Sens. Actuators, B 231, 529-538 (2016).

40. Gomes, J., Rodrigues, J. J., Rabêlo, R. A., Kumar, N. \& Kozlov, S. IoT-enabled gas sensors: technologies, applications, and opportunities. J. Sens. Actuator Netw. 8, 57 (2019).

41. Deng, Y. et al. Black phosphorus-monolayer $\mathrm{MoS}_{2}$ van der Waals heterojunction p-n diode. ACS Nano 8, 8292-8299 (2014).

42. Youngblood, N., Chen, C., Koester, S. J. \& Li, M. Waveguide-integrated black phosphorus photodetector with high responsivity and low dark current. Nat. Photonics 9, 247-252 (2015).

43. Yuan, H. et al. Polarization-sensitive broadband photodetector using a black phosphorus vertical p-n junction. Nat. Nanotechnol. 10, 707-713 (2015).

44. Guo, Q. et al. Black phosphorus mid-infrared photodetectors with high gain. Nano Lett. 16, 4648-4655 (2016).

45. Huang, M. et al. Broadband black-phosphorus photodetectors with high responsivity. Adv. Mat. 28, 3481-3485 (2016).

46. Chen, X. et al. Widely tunable black phosphorus mid-infrared photodetector. Nat. Commun. 8, 1-7 (2017). 
47. Bullock, J. et al. Polarization-resolved black phosphorus/molybdenum disulfide mid-wave infrared photodiodes with high detectivity at room temperature. Nat. Photonics 12, 601607 (2018).

48. Amani, M., Regan, E., Bullock, J., Ahn, G. H. \& Javey, A. Mid-wave infrared photoconductors based on black phosphorus-arsenic alloys. ACS Nano 11, 11724-11731 (2017). 
a

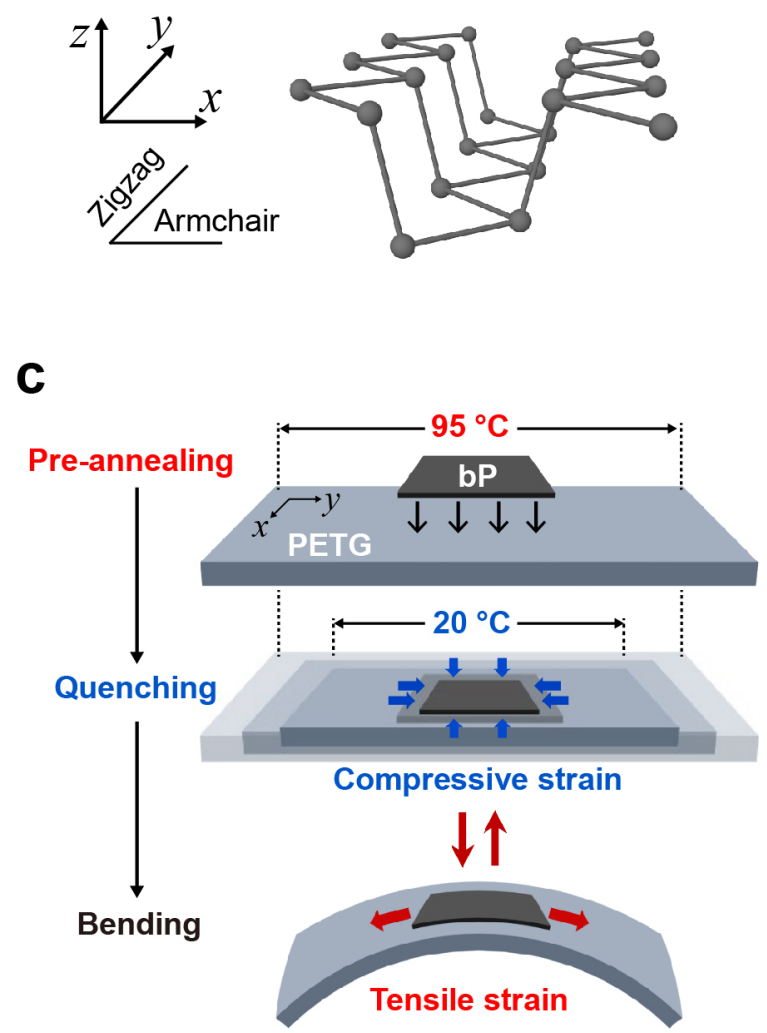

b

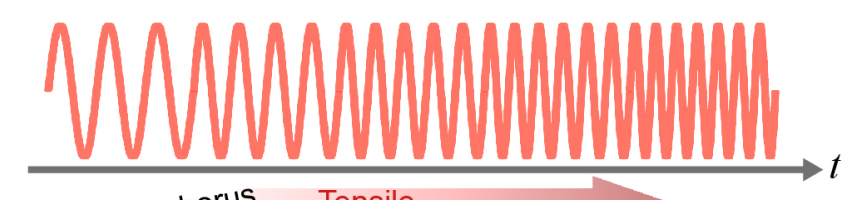

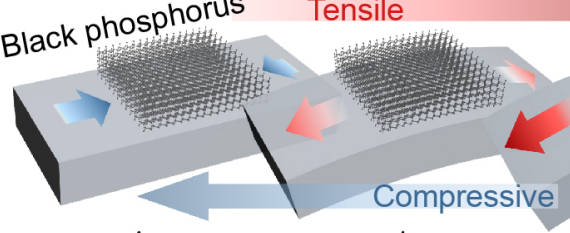

$\stackrel{\longrightarrow}{\longrightarrow} v$
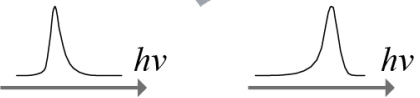

d

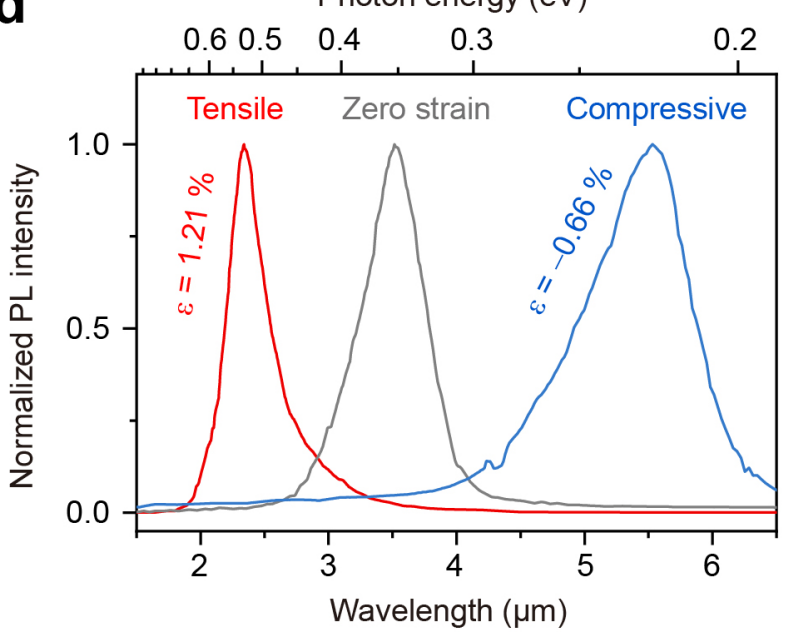

Fig. 1 | Strain-tunable bandgap in bP. a, Schematic illustrating the anisotropic crystal structure of bP along the armchair (AC) and zigzag (ZZ) directions. b, Schematic representation of actively tunable bandgap of bP using strain. Compressive and tensile strains induce redshift and blueshift of the bP bandgap, respectively. c, Schematic diagram illustrating strain application in bP. Compressive strain is applied by thermal expansion mismatch between bP and the substrate and tensile strain is applied by mechanical bending of the substrate. d, Normalized PL spectra of asexfoliated $\mathrm{bP}$, and $\mathrm{bP}$ under compressive strain (0.66\% along $\mathrm{ZZ})$ and $\mathrm{bP}$ under tensile strain $(1.21 \%$ along ZZ). BP thickness is $20 \mathrm{~nm}$, and the excitation light is polarized in the AC direction at an 
incident power of $22.5 \mathrm{~W} / \mathrm{cm}^{2}$ (there is no laser-induced thermal effect on the strain conditions of bP). Tensile strain is applied in the ZZ direction of the bP on the PETG substrate. 

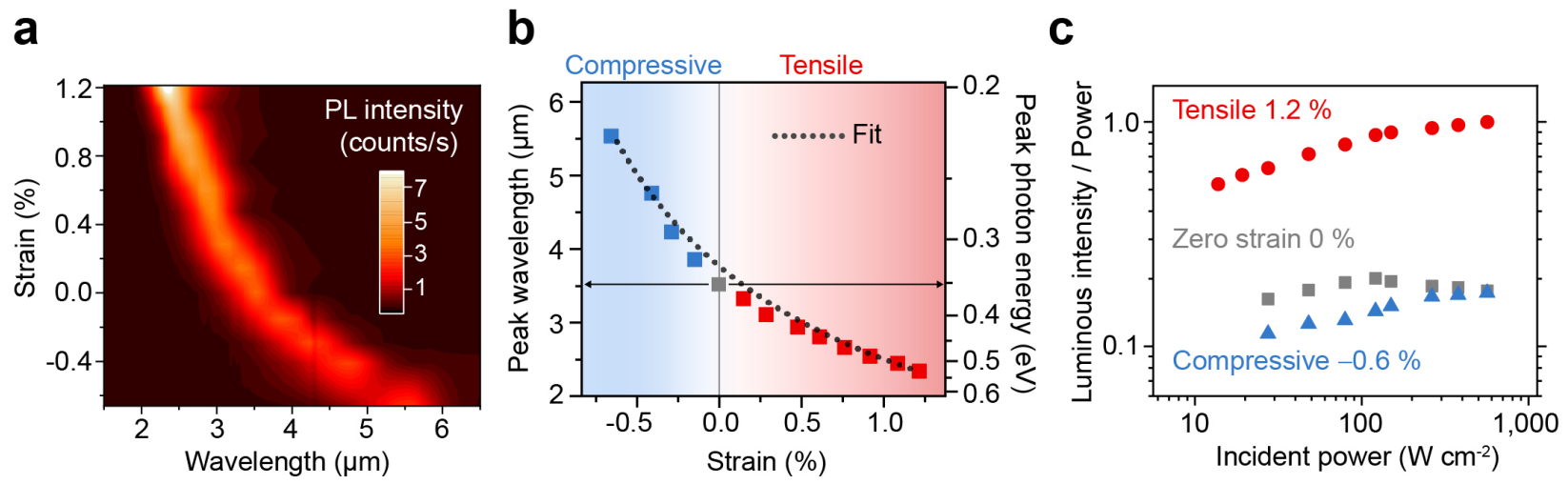

Fig. 2 | Strain-induced bandgap modification in bP. a, PL spectra of bP as a function of strain along the ZZ (y) direction. b, Strain-dependent PL spectral peak shift in bP. The fit indicates that the peak modulation rate is $1.70 \mu \mathrm{m}$ wavelength shift per percent of strain, covering from 5.5 to $2.3 \mu \mathrm{m}$. Laser-induced thermal effects on strain are precluded by using low excitation power, where the PL spectral peak is constant with increasing laser power density (Extended Data Fig. 2b). c, Integrated PL intensity normalized by incident power as a function of power density for bP under different strain conditions. Note that bP thickness is $20 \mathrm{~nm}$ and the PETG substrate is used in the above measurements. We also demonstrated reversible and repeatable bandgap tuning, which indicates that there is no mechanical slip between bP and the substrate over multiple cycles (Extended Data Fig. 2e). 
a

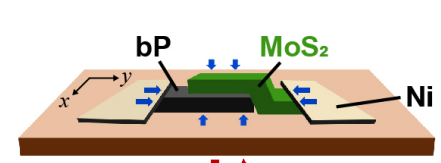

Polyimide $\downarrow \uparrow$

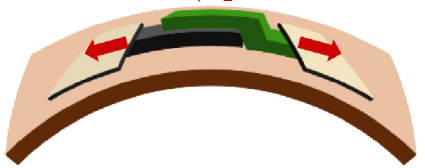

d
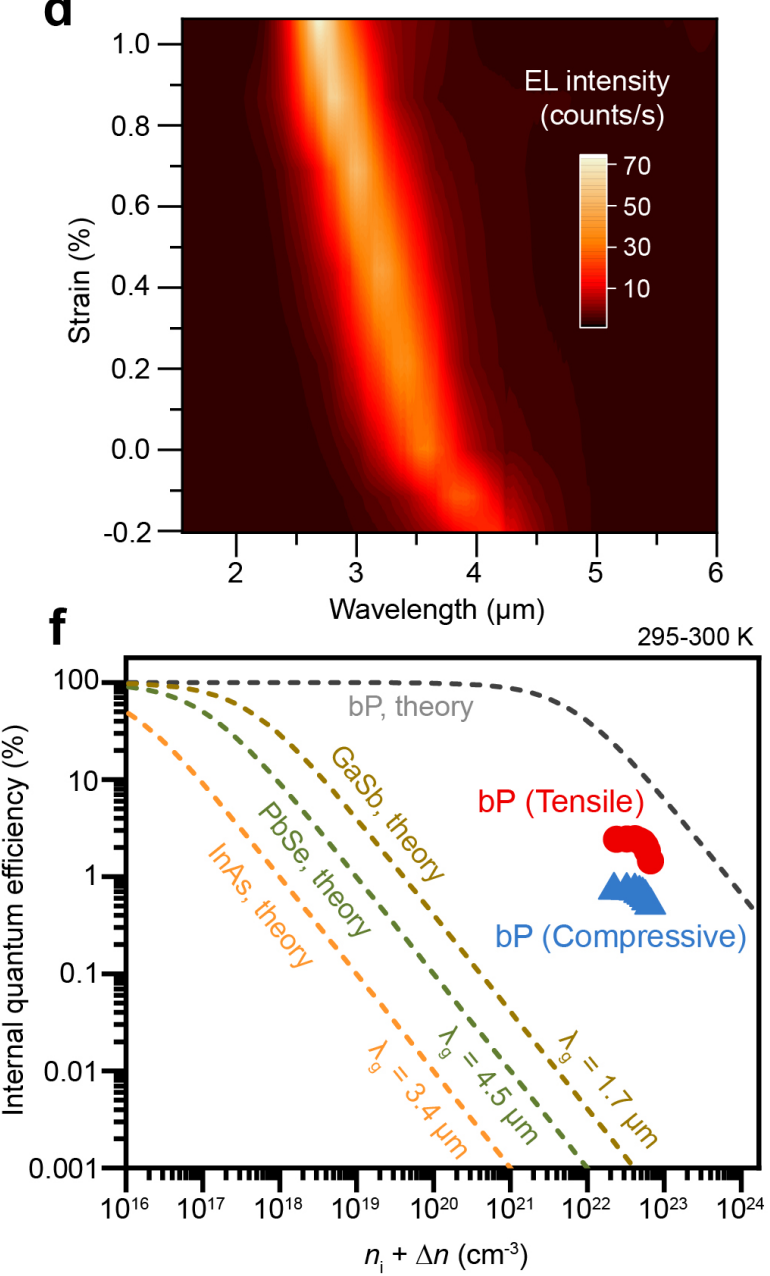

b

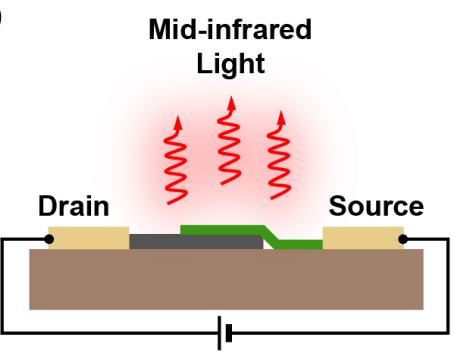

C

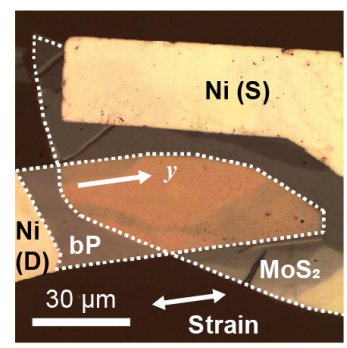

e

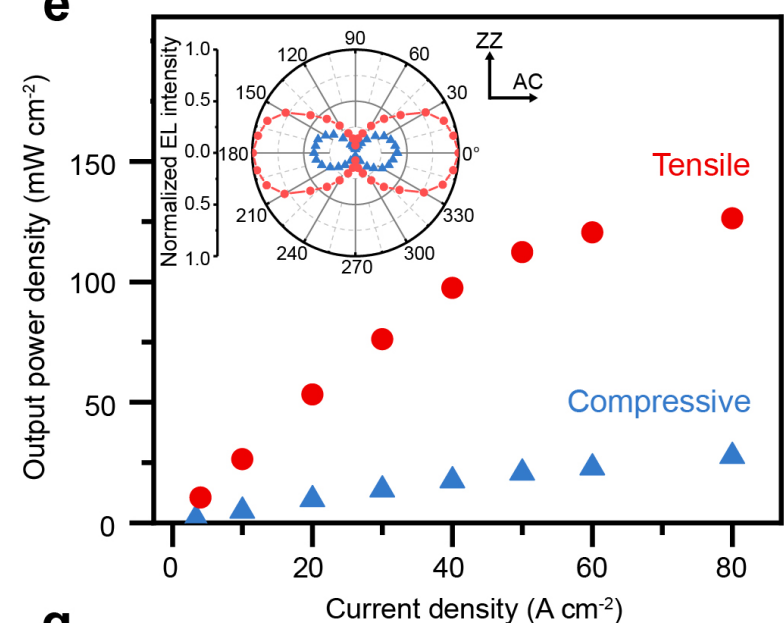

$\mathbf{g}$

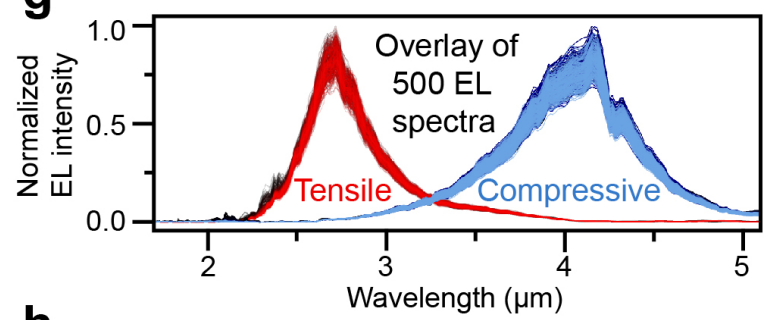

h

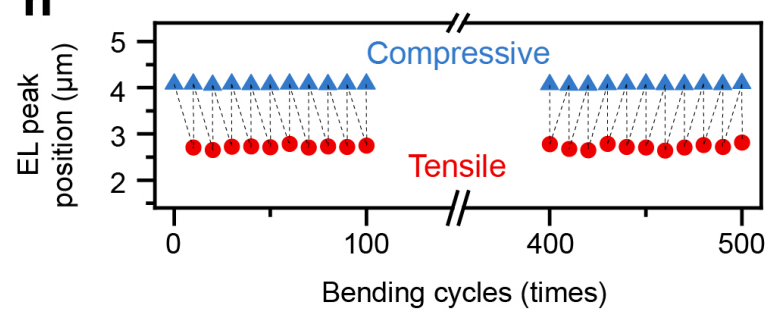

Fig. 3 | Strain-tunable MWIR LEDs based on bP/MoS2 heterostructure. a, Schematic of a strain-tunable bP/MoS 2 LED. b, Schematic of the device architecture, showing MWIR electroluminescence under forward bias across the $\mathrm{bP} / \mathrm{MoS}_{2}$ heterojunction on the polyimide substrate. c, Optical micrograph of a representative device. d, Strain-dependent EL spectra. e, 
Light-current characteristics under $0.20 \%$ compressive and $1.06 \%$ of tensile strain. Inset: integrated EL intensity as a function of emission polarization angle. f, Theoretical Auger recombination-limited internal $\mathrm{QY}$ for $\mathrm{InAs}, \mathrm{PbSe}, \mathrm{GaSb}$, and $\mathrm{bP}$ at room temperature and the measured internal quantum efficiency of bP device shown in panel e. g, Overlay of 500 EL spectra and $\mathbf{h}$, EL spectral peak shift observed in a bP-LED, during 500 cycles of bending $(1.06 \pm 0.05 \%$, tensile) and relaxation (Supplementary Video 1). Note that bP thickness of the device is $20 \mathrm{~nm}$ and all the EL measurements were performed at a current density of $20 \mathrm{~A} / \mathrm{cm}^{2}$. 
a

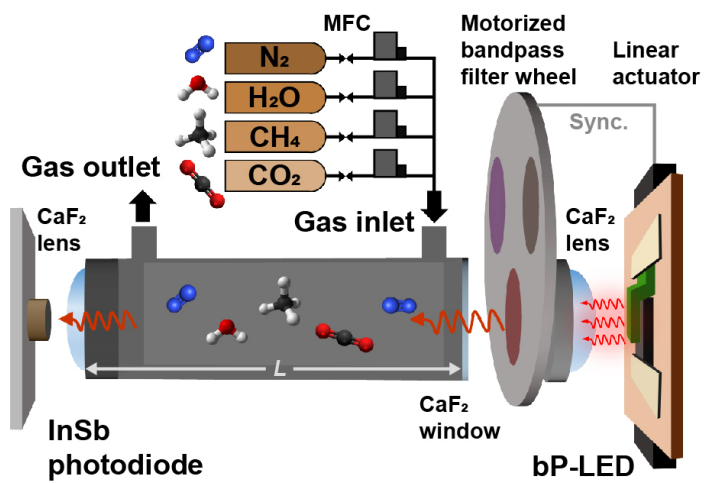

d

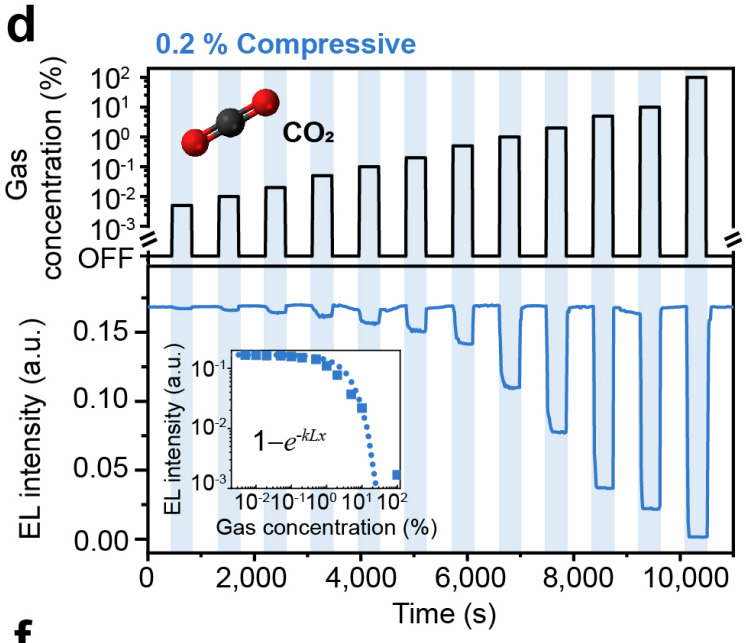

b

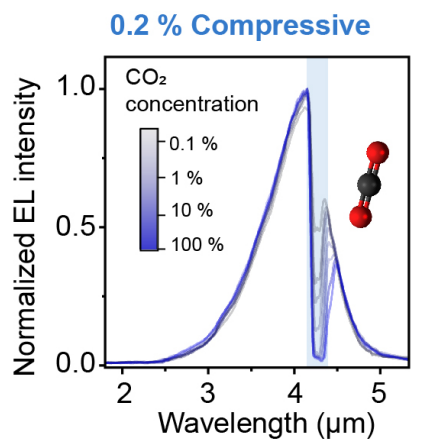

e
C
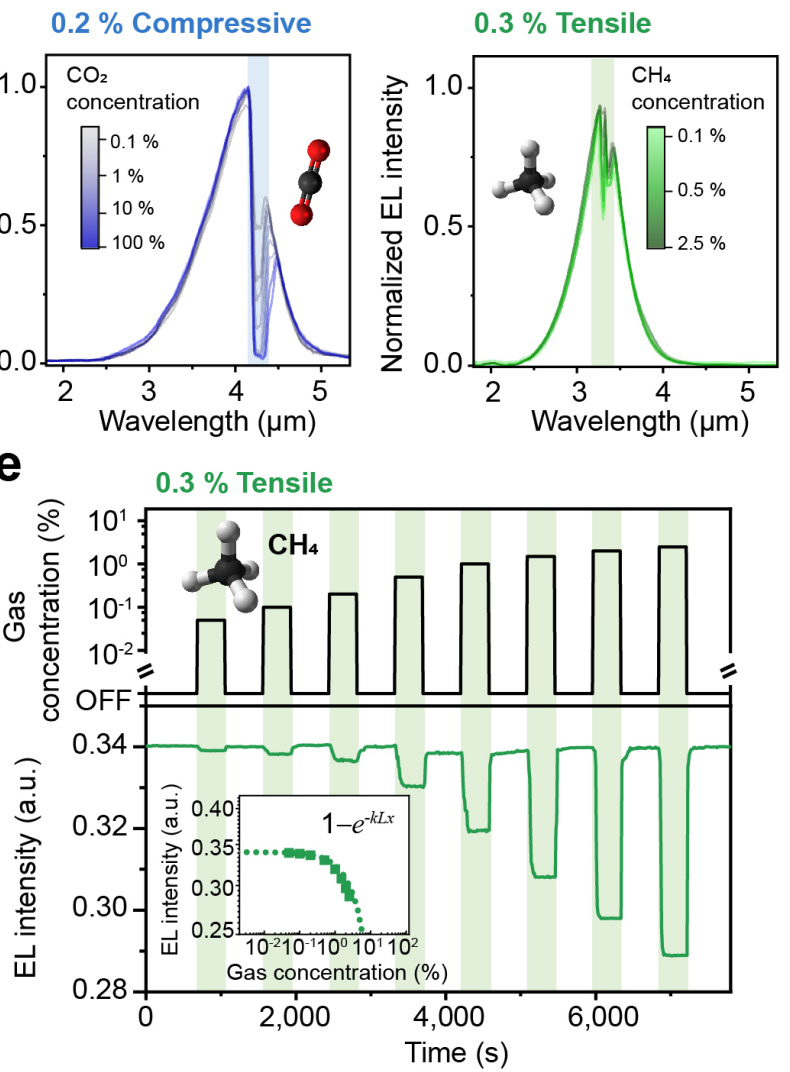

$\mathbf{f}$

$2 \%$

$\mathrm{CH}_{4}$ only

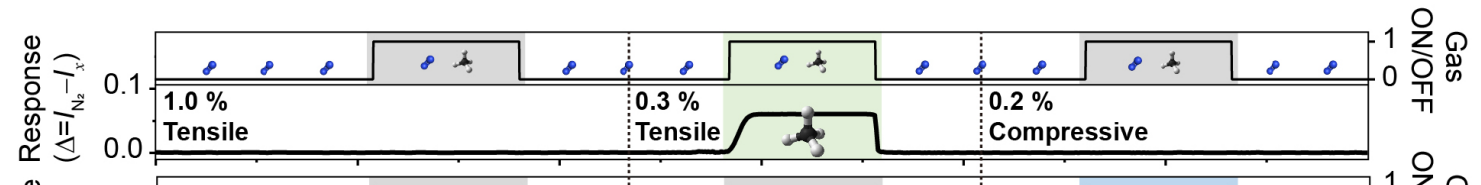

$0.2 \%$

$\mathrm{CO}_{2}$ only

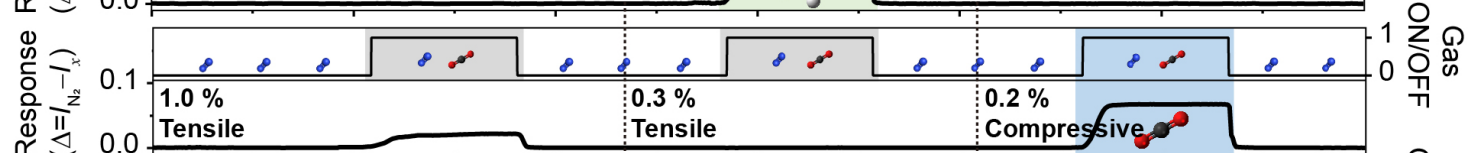

$20 \%$

R.H. only

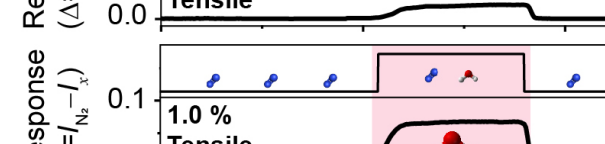

Compressivege

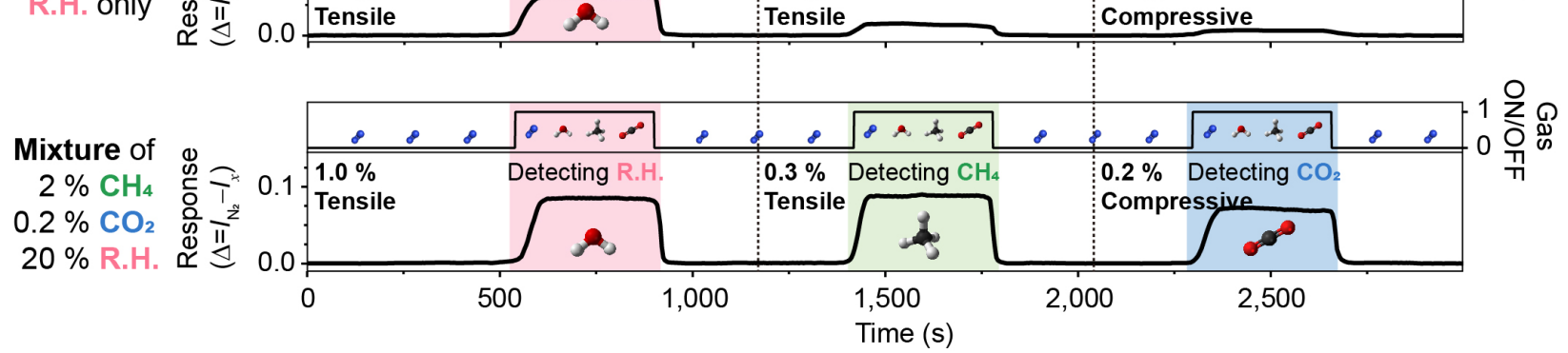

Fig. 4 | NDIR gas sensing using strain-tunable LEDs. a, Schematic diagram of the NDIR gas sensing system using a strain-tunable bP-LED. b,c, Normalized EL spectra of a bP-LED (0.2 \%, 
compressive) in the presence of $\mathrm{CO}_{2}$ gas (b) and bP-LED (0.3\%, tensile) in the presence of $\mathrm{CH}_{4}$ gas (c) at different concentrations. d,e, Sensor response characteristics using bP-LED $(0.2 \%$, compressive) for varying concentrations of $\mathrm{CO}_{2}$ pulses (d) and bP-LED $(0.3 \%$, tensile) for varying concentrations of $\mathrm{CH}_{4}$ pulses (e). Inset: the data fit using Beer-Lambert law model. f, Real-time multiplexed sensing of the individual target gas, from a gas mixture consisting of $\mathrm{H}_{2} \mathrm{O}(20 \%$ R.H.), $\mathrm{CH}_{4}(2 \%)$, and $\mathrm{CO}_{2}(0.2 \%)$, using bP-LED under $1.0 \%$ tensile, $0.3 \%$ tensile, and $0.2 \%$ compressive strain, respectively. Responses from individual gases $\left(\mathrm{CH}_{4}, \mathrm{CO}_{2}\right.$, and $\left.\mathrm{H}_{2} \mathrm{O}\right)$ are measured under different strains to characterize the selectivity of multiplexed gas sensing system. Note that the EL intensity (d-e) is the value recorded by the InSb photodiode on the opposite end of the gas cell. 


\section{Methods}

Sample preparation. BP crystals (Smart Elements) were mechanically exfoliated onto a polydimethylsiloxane (PDMS) substrate $\left(1 \times 1 \mathrm{~cm}^{2}\right)$ with PVC tape (SPV224, Nitto). Prior to the transfer process, the crystal orientation of bP was identified by polarization-resolved Raman spectroscopy, following the method described in previous work ${ }^{49}$. The transfer process used here is similar to the dry-transfer process described previously ${ }^{50}$. It was performed in a modified optical microscope setup (Labophot, Nikon) with the sample stage replaced by a hotplate. A PETG substrate $\left(2.54 \mathrm{~mm}\right.$ thick, $\left.3 \times 3 \mathrm{~cm}^{2}\right)$ was placed on a hotplate at $95^{\circ} \mathrm{C}$. The exfoliated bP on PDMS stamp was positioned upside down and aligned with the center of the PETG substrate. The stamp was slowly brought into contact with the thermally expanded PETG and bP was transferred at $95{ }^{\circ} \mathrm{C}$. The bP-transferred PETG substrate was immediately subjected to rapid thermal quenching, bring it down to room temperature. Due to the CTE mismatch and the rapid thermal quenching process, bP is subjected to biaxial compressive strain. After the transfer, the prepared bP sample with biaxial compressive strain was loaded into the two-point bending apparatus (Extended Data Fig. 1a) inside the optical cryostat (ST-100, Janis). All processes were performed inside a $\mathrm{N}_{2}$ purged glovebox (830-ABC, Plas-Labs) or in environments with minimum moisture and light exposure, to avoid any ambient atmosphere-induced bP oxidation.

Device fabrication. For LED fabrication, electronic-grade polyimide $\left(0.5 \mathrm{~mm}\right.$ thick, $\left.1 \times 1 \mathrm{~cm}^{2}\right)$ was used as a substrate instead of PETG due to its compatibility with electron-beam lithography processes. The exfoliation and transfer process of $\mathrm{bP}$ and $\mathrm{MoS}_{2}$ followed the same procedure as mentioned in the sample preparation section. BP (thickness $\sim 15-22 \mathrm{~nm}$ ) was first transferred onto the polyimide substrate at $180{ }^{\circ} \mathrm{C}$, followed by rapid quenching to room temperature. 
Subsequently, $\mathrm{MoS}_{2}$ (thickness $\sim 10-15 \mathrm{~nm}$, SPI Supplies) exfoliated on a PDMS stamp, was transferred on top of the bP at room temperature to form an electron contact to the bP. Source and drain electrodes were patterned by conventional electron-beam lithography using poly(methyl methacrylate) (PMMA) C4 resist (baked at $130^{\circ} \mathrm{C}$ ) and thermal evaporation of $40 \mathrm{~nm} \mathrm{Ni}$.

For photoconductor fabrication, bright field-mode photolithography was used, followed by subtractive wet chemical etching, where the opaque portion of the photomask is the desired electrode patterns. First, $100 \mathrm{~nm} \mathrm{Au}$ was evaporated on the bP-transferred PETG, which was prepared by the methods mentioned earlier. S1818 (Shipley Microposit) was coated on bPtransferred PETG and the sample was baked at $80{ }^{\circ} \mathrm{C}$ for $3 \mathrm{~min}$, which is below the glass transition temperature of PETG $\left(90-95{ }^{\circ} \mathrm{C}\right)$. After the g-line exposure using a bright field photomask, followed by the development process (MF-26A, Microchem), the exposed Au region was completely etched using a gold etchant (potassium iodide, Transene). Finally, the residual photoresist layer was removed by flood exposure followed by the development process.

Optical characterization. All optical measurements were performed in a customized cryostat (ST100, Janis) with a $\mathrm{CaF}_{2}$ window, which was evacuated to a base pressure $<10^{-5}$ Torr. A two-point bending apparatus was installed with the electric actuator inside the cryostat, so that the amount of uniaxial tensile strain can be simultaneously controlled during the measurement. For PL and EL measurements, a 15x reflective objective was installed at the auxiliary entrance port of the FT-IR (iS50, Thermo Fisher), collecting the sample signal and sending it to the FT-IR spectrometer equipped with a $\mathrm{CaF}_{2}$ beam splitter and a liquid $\mathrm{N}_{2}$-cooled $\mathrm{HgCdTe}$ (MCT) detector. A laser diode (Thorlabs, $\lambda=638 \mathrm{~nm}$ ) and a laser diode controller (LDC500, Thorlabs) were used as the excitation source for the PL measurements. A mirror was installed at the obscured center of the reflective 
objective to reflect the focused excitation beam onto the sample. The polarization of the excitation laser was aligned to be parallel to the $\mathrm{AC}$ orientation of $\mathrm{bP}$ by rotating the polarization plane using a linear polarizer followed by a half-wave plate. For EL measurement, a voltage controlled current source (CS580, Stanford Research Systems) was used to apply a forward current bias. The instrument response function of the setup accounted for different wavelengths and polarizations, following the same procedure as described in our previous work ${ }^{47}$. For the spectrum calculation process, double modulation was used to separate the PL/EL signal from the thermal background in the modulated interferogram, which was obtained through a lock-in amplifier (SR865, Stanford Research Systems) and low-noise current preamplifier (SR560, Stanford Research Systems). Details of the PL and EL measurement setup are further described in Extended Data Fig. 8.

Photodetector characterization was performed at the auxiliary exit port of the FT-IR, where the bP photoconductor was excited by modulated $1000 \mathrm{~K}$ blackbody illumination. The device was biased at $100 \mathrm{mV}$. The generated photocurrent was amplified by the low-noise current preamplifier (SR570, Stanford Research Systems) and sent to the external detector interface of the FT-IR system, in order to resolve the full spectral responsivity. $\mathrm{A} \mathrm{BaF}_{2}$ linear polarizer was placed at the unpolarized excitation path for the polarization resolved photoresponse measurements. For temporal photoresponse measurements, a laser diode (Thorlabs, $\lambda=1650 \mathrm{~nm}$ ) was modulated by a square wave generated using a function generator (81150A, Agilent). The FT-IR system was calibrated following the procedure described in our previous work ${ }^{47}$. An internal deuteratedtriglycine sulfate (DTGS) detector was used to measure the relative spectral intensity of the excitation. A NIST-calibrated Ge photodiode (Thorlabs) and InSb photodiode (Teledyne Judson Technologies) were used as reference to determine the absolute responsivity of our bP 
photoconductors, as a function of wavelength. Raman measurement was performed in a commercial setup (inVia, Renishaw) with a $532 \mathrm{~nm}$ laser excitation, which was linearly polarized along $45^{\circ}$ with respect to the $\mathrm{AC}$ and $\mathrm{ZZ}$ orientation of $\mathrm{bP}$, in order to optimize the signal intensity from all three characteristic Raman modes $\left(A_{\mathrm{g}}^{1}, B_{2 \mathrm{~g}}\right.$, and $\left.A_{\mathrm{g}}^{2}\right)$ at the same time. For both PL and Raman measurements, the lowest excitation power that gave a perceptible signal-to-noise ratio (SNR) was used, to prevent any localized heat-induced strain effects in bP. The effects of laserinduced heating on the strain conditions of bP is characterized in Extended Data Fig. $2 \mathrm{~b}$.

NDIR gas sensing characterization. In the NDIR gas sensing setup, target gases and diluent gas $\left(\mathrm{N}_{2}\right)$ were injected into an enclosed gas cell, which was illuminated by the bP-LED at one end. At the opposite end, a commercial InSb photodiode was used to measure the light intensity passing through the gas cell (Fig. 4a). EL spectra (Fig. 4b,c) were measured by the internal MCT detector in the FT-IR system, under the constant flow of a target gas at various concentrations. NDIR gas sensor responses (Fig. 4d,e and Extended Data Fig. 5b,c) were characterized by a commercial liquid $\mathrm{N}_{2}$-cooled InSb photodiode to a sequence of target gas pulses and purging cycles of $\mathrm{N}_{2}$ gas. A pair of $\mathrm{CaF}_{2}$ plano-convex lenses or $15 \mathrm{x}$ reflective objectives were used to improve the dynamic range of the measurements. A motorized filter wheel (FW102C, Thorlabs) was used with the bandpass filters for $\mathrm{H}_{2} \mathrm{O}$ (Center wavelength $(\mathrm{CWL})=2700 \mathrm{~nm}$, Iridian), $\mathrm{CH}_{4}(\mathrm{CWL}=3330 \mathrm{~nm}$, Thorlabs), and $\mathrm{CO}_{2}(\mathrm{CWL}=4260 \mathrm{~nm}$, Thorlabs $)$ detection. The bP-LED was modulated at $1 \mathrm{kHz}$ and the generated photocurrent for the InSb photodiode was amplified using a low-noise current preamplifier (Stanford Research Systems) and measured by the lock-in amplifier (Stanford Research Systems). $\mathrm{CH}_{4}$ gas cylinder $(2.5 \%), \mathrm{CO}_{2}$ gas cylinder (> 99.9\%), and moisturecontaining $\mathrm{N}_{2}$ gas cylinder were connected to individual mass flow controllers (Alicat) to mix and 
deliver the gas pulse with precise concentrations. Humidity inside the gas cell was monitored by a commercial digital humidity sensor (SHT2x, Sensirion). At room temperature (292-295 K), the current density was kept at $20 \mathrm{~A} / \mathrm{cm}^{2}$ for all measurements using $20 \mathrm{~nm}$ bP-LED. It is noted that the baseline current $\left(I_{N_{2}}\right)$ was different when the system was configured to sense the different gases. This is attributed to the changing emission intensity from the bP-LED subjected to applied strain conditions, different transmission values of the filters used for each gas detection, and the varying spectral response of the InSb photodiode.

BP for strain application. Exfoliated bP flakes with cracks or wrinkles did not show any straininduced bandgap modification with our methods described in this work. Significant efforts were thus made during the sample preparation and fabrication to avoid cracks/wrinkles within bP flakes. We mainly focused on the demonstration of bandgap tuning and its device applications for bP with thicknesses of $15-22 \mathrm{~nm}$. For this thickness range, carrier transport is less susceptible to environment than thinner bP, while strain-induced bandgap tuning has a higher yield than its thicker counterpart. We found that the strain-induced bandgap modification using our method started to become less effective for bP thicker than $22 \mathrm{~nm}$, possibly due to slippage between the $\mathrm{bP}$ and the substrate. On the other hand, bP thinner than $15 \mathrm{~nm}$ had a lower yield of bandgap tuning by strain, which could be attributed to the difficulty in processing thinner bP without cracks, and accelerated oxidation in thinner bP from layer-by-layer thinning ${ }^{51}$. Since the thickness characterization of bP was challenging with direct atomic force microscopy (AFM) on a soft polymer substrate, we used the method described in the previous work ${ }^{20}$ to identify the bP with a suitable thickness. 
Compressive strain application. Negative bending (i.e. in opposite direction to that used for tensile strain) was first used with the goal of applying compressive strain. However, this induced decoupling of bP from the substrate and buckling of bP possibly due to the Poisson effect. Therefore, to apply compressive strain, thermal expansion mismatch between bP and substrate was used instead. PETG is the material that was selected as a substrate as it was found to have favorable properties compared to other polymers (e.g. Polypropylene (PP), Polyethylene (PE), Ethylenevinyl acetate (EVA), Polycarbonate (PC), Polyvinylidene fluoride (PVDF), PDMS, Nylon, Polyvinyl chloride (PVC), Polystyrene (PS), and Teflon). A transfer temperature of $95^{\circ} \mathrm{C}$ was used, which is slightly higher than the glass transition temperature of PETG. The CTE of PETG was experimentally measured to be $80.5 \pm 2.5 \mathrm{ppm} /{ }^{\circ} \mathrm{C}$ and $520 \pm 32 \mathrm{ppm} /{ }^{\circ} \mathrm{C}$, over the temperature range of $20-90{ }^{\circ} \mathrm{C}$ and $90-95{ }^{\circ} \mathrm{C}$, respectively, indicating that the thermal expansion rate rapidly increases at the glass transition temperature ${ }^{52}$. It was found that the higher transfer temperature ( $T$ $=100{ }^{\circ} \mathrm{C}$ ) resulted in wrinkles and delamination of bP due to the low adhesion and excessive compressive strain from the substrate ${ }^{53}$ and did not contribute to larger modulation range by strain. Therefore, we did not use transfer temperatures in excess of $95^{\circ} \mathrm{C}$. BP has anisotropic CTEs of 33 (AC) and $22(\mathrm{ZZ}) \mathrm{ppm} /{ }^{\circ} \mathrm{C}^{54}$. The compressive strain applied in bP due to the CTE mismatch can thus be calculated as follows:

$$
\varepsilon\left(T_{\text {tr }}\right)=\int_{20^{\circ} \mathrm{C}}^{T_{\text {tr }}} \alpha_{\text {sub }}(T) d T-\int_{20^{\circ} \mathrm{C}}^{T_{\text {tr }}} \alpha_{\mathrm{bP}}(T) d T
$$

where $T_{\text {tr }}$ is the transfer temperature of bP onto the substrate, $\alpha_{\text {sub }}$ is the CTE of the substrate, and $\alpha_{\mathrm{bP}}$ is the CTE of bP. The compressive strain resulting from this quenching process was $0.58 \%$ 
in the $\mathrm{AC}$ direction and $0.66 \%$ in the $\mathrm{ZZ}$ direction. The strain in $\mathrm{AC}(x)$ direction can be obtained using the Poisson's ratio of bP.

Substrate for device fabrication. The chemical instability of bP has been a main roadblock in demonstrating strain-tunable optoelectronic devices based on bP. Here, we developed a method and procedure that are compatible with conventional lithography, while maintaining the material composition and device performance of bP. Although the polyimide has a lower CTE (experimentally measured to be $34.8 \pm 1.5 \mathrm{ppm} /{ }^{\circ} \mathrm{C}$ at $20-200{ }^{\circ} \mathrm{C}$ ) and is softer than PETG, it was used for bP/MoS $\mathrm{M}_{2}$ LEDs for following reasons. Polyimide is chemically stable, resistant to high temperature $\left(>100{ }^{\circ} \mathrm{C}\right)$, and compatible with conventional lithography processes and organic solvent processing. These enable electrode patterning with a desired shape and higher spatial resolution, which reduces the series resistance of the LEDs. In addition, the electronic-grade polyimide we use has a higher thermal conductivity than PETG, which is advantageous for repeated operations over long periods of time without performance degradation due to thermal failure and charging issues. Note that compressive strain in $\mathrm{bP} / \mathrm{MoS}_{2}$ LEDs is not completely released during the high temperature measurement (Extended Data Fig. 4) because of the low CTE of the polyimide substrate.

Preserving strain and stability during fabrication. The built-in strain between a $2 \mathrm{D}$ transition metal dichalcogenide (TMDC) monolayer and the substrate can be released by solvent evaporation-mediated decoupling process ${ }^{55}$. However, we find that the bP-MoS 2 LED in our work still preserved partial compressive strain after the fabrication. This can be attributed to the source/drain electrodes and $\mathrm{MoS}_{2}$ being on top of the bP, which serve as a clamp to prevent the complete release of the biaxial compressive strain between bP and the polyimide substrate. During 
the lift-off process, acetone evaporation was avoided and toluene was used instead of isopropyl alcohol (IPA), which prevents the release the built-in strain in 2D materials due to evaporation. For the fabrication of bP photoconductor, the photoresist developer (MF-26A) and the gold etchant (potassium iodide) did not affect the biaxial compressive strain in bP, which was mostly maintained after the fabrication was completed. Moreover, the gold film evaporated on bP could have functioned as a clamping agent, adding/holding the biaxial compressive strain in bP. It is reported in our earlier work that metal evaporation on 2D materials results in the biaxial compressive strain due to the thermal expansion mismatch between the metal and material ${ }^{56,57}$. During photoconductor fabrication, except the 5s of g-line exposure, visible light exposure of bP was minimized to prevent photooxidation. In agreement with the previous work ${ }^{58}$, we observed that the gold etchant (potassium iodide) and DI water alone did not induce bP oxidation and the device performance was not degraded during the fabrication. It is understood that water, oxygen, and visible light are simultaneously required for oxidation of bP.

Measurement accuracy. Rather than using extinction spectra to estimate the bandgap, we instead used IRPL spectroscopy to characterize the bandgap modification in bP under strain. For EL and PL measurement, it is important to note that the measurement setup was calibrated for each value of strain, to capture the absolute intensity of light emission from the non-planar surface. This was required to exclude the possibility of focal plane drift upon bending of the substrate. For the PL measurements, the excitation laser spot size $\left(\sim 1600 \mu \mathrm{m}^{2}\right)$ was maintained to be slightly smaller than the size of the bP flake. At each measurement, the polarization of excitation with respect to the bP crystal orientation was verified by comparing the results between rotating the polarizer in the excitation path and rotating the sample with respect to a fixed laser polarization. BP absorption 
at the PL excitation wavelength $(638 \mathrm{~nm})$ was observed to be weakly dependent on the polarization angle, while it is expected to have the highest anisotropy near the absorption edge ${ }^{43}$. For photoconductor characterization, a fixed voltage bias of $V_{\mathrm{ds}}=100 \mathrm{mV}$ (smaller than the bP bandgap) was used for all measurements to maintain the responsivity of the photoconductor in a linear regime.

LED efficiency calibration. To quantify the light emission efficiency of our bP-LEDs, we used $4.05 \mu \mathrm{m}$ quantum cascade laser (QF4050T1, Thorlabs) and an infrared reflectance standard (Labsphere). The laser output power was measured using a high sensitivity thermal power sensor (Ophir) at the focal plane of the objective. The laser signal intensity reflected by the Lambertian reflectance standard at the focal plane was measured by the FT-IR under the same measurement conditions. Using the ratio of the output power measured from the power sensor to the signal intensity measured by the MCT detector in the FT-IR, we can convert the emission intensity from bP-LED measured by the FT-IR into output power. The instrument response function (IRF) of the MCT detector is taken into consideration when accounting for the difference between the laser and bP-LED emission wavelengths. In addition, since the reflective objective has a fixed collection angle, the angular collection factor was calculated to account for the difference between the angular distribution of emission of the bP-LEDs and of the Lambertian emitter. The output power of our bP-LED is then calculated by:

$$
\text { Power }_{\text {bPLED }}=\text { Intensity }_{\text {bPLED (FTIR) }} \times \frac{\text { Power }_{\text {laser (power meter) }}}{\text { Intensity }_{\text {laser (FTIR) }}} \times \frac{\operatorname{IRF~}\left(\lambda_{\text {bPLED }}\right)}{\operatorname{IRF}(\lambda=4.05 \mu \mathrm{m})} \times \frac{\text { Angular collection }_{\text {bPLED }}}{\text { Angular collection }_{\text {laser }}}
$$

where $\operatorname{IRF}\left(\lambda_{\mathrm{bPLD}}\right)$ and $\operatorname{IRF}(\lambda=4.05 \mu \mathrm{m})$ are the instrument response function of the MCT detector at the bP-LED emission wavelength and $4.05 \mu \mathrm{m}$, respectively, and Angular collection ${ }_{\mathrm{bPLED}}$ and 
Angular collection ${ }_{\text {laser }}$ are the collected signal with the objective lens when the on-axis $\left(0^{\circ}\right)$ emission intensity is assumed to be 1 . Note that to estimate the instantaneous carrier concentration in the bP-LED at different current densities in Fig. 3f, we assume that the carriers are uniformly distributed in the bP volume.

Strain-tunable photodetection. As expected from the highly polarization-dependent absorption of $\mathrm{bP}^{59}$, we observed the highest photoresponse when light was polarized in the AC direction and the lowest photoresponse when it was polarized in the ZZ direction (Extended Data Fig. 6e). The $I-V$ characteristics of the photoconductors under compressive and tensile strain conditions displayed no obvious difference (Extended Data Fig. 6f). Under compressive strain, the rise and fall times of the photoconductors were measured to be $18.6 \mu$ s and $16.3 \mu \mathrm{s}$, respectively (Extended Data Fig. 6g). Under tensile strain, they showed similar values of $18.7 \mu$ s and $16.6 \mu$ s, respectively. The noise current density did not show any significant variation between compressive strain and tensile strain conditions, and the low frequency noise dominated by the contribution from $1 / f$ noise (Extended Data Fig. 7a).

To estimate the performance of our tunable photoconductor, the specific detectivity $\left(D^{*}\right)$ was calculated as $D^{*}=\sqrt{A \Delta f} / N E P$ where $A$ is the device area, $\Delta f$ is the bandwidth, and $N E P$ is the noise equivalent power. The bandwidth of the photoconductor is measured to be $10 \mathrm{kHz}$ under both compressive and tensile strain (Extended Data Fig. 7b). A detailed discussion of the process we used to estimate $N E P$ is provided in the latter part of Methods, where we utilized the experimentally extracted noise current to avoid an overestimation of $D^{*}$. Given a constant device area and integration time, the optimum bP thickness that maximizes the $D^{*}$ of a bP photoconductor is reported to be $\sim 25-35 \mathrm{~nm}$, which originates from the balance between increasing absorption 
from greater thickness and reducing noise in fewer layers ${ }^{48}$. By applying strain to a photoconductor based on bP with a thickness close to this range, we can extend its spectral photoresponse while preserving optimal device performance. The detectivity could be further improved by enhancing the light collection efficiency of the device and/or implementing a gate bias to compensate for background doping ${ }^{47,48}$.

Noise equivalent power (NEP) calculation. NEP is the optical power that gives a SNR of unity for a given measurement bandwidth of a photodetector. It is determined by calculating the photocurrent $\left(I_{\mathrm{ph}}=\operatorname{Responsivity~}\left(R_{\lambda}\right) \times N E P\right)$ when $\mathrm{SNR}=1=I_{\mathrm{ph}}^{2} /<i_{\mathrm{n}}^{2}>$. Since the total noise current is mainly determined by the sum of the contributions from shot noise and Johnson noise, we can formulate it as follows: $<i_{\mathrm{n}}^{2}>=2 e\left(I_{\mathrm{ph}}+I_{\mathrm{dark}}\right) \Delta f+\frac{4 k_{\mathrm{B}} T}{R} \Delta f$, where $e$ is the electron charge, $\Delta f$ is the bandwidth, $k_{\mathrm{B}}$ is the Boltzmann constant, $T$ is the temperature, and $R$ is the dark resistance which is determined from the $I-V$ characteristics of the photoconductor (Extended Data Fig. 6f). Solving the equation $\left(I_{\mathrm{ph}}^{2}-2 e\left(I_{\mathrm{ph}}+I_{\mathrm{dark}}\right) \Delta f-\frac{4 k_{\mathrm{B}} T}{R} \Delta f=0\right)$ gives the value of $I_{\mathrm{ph}}$, where all the other parameters in the equation are obtained from the experimental data. Therefore, NEP is calculated as $6.54476 \times 10^{-11} / R_{\lambda}^{\text {compressive }}$ for compressive strain and $6.69445 \times 10^{-11} / R_{\lambda}^{\text {tensile }}$ for tensile strain. 
49. Wu, J., Mao, N., Xie, L., Xu, H. \& Zhang, J. Identifying the crystalline orientation of black phosphorus using angle-resolved polarized Raman spectroscopy. Angew. Chem. Int. Ed. 54, 2366-2369 (2015).

50. Castellanos-Gomez, A. et al. Deterministic transfer of two-dimensional materials by alldry viscoelastic stamping. 2D Mater. 1, 011002 (2014).

51. Favron, A. et al. Photooxidation and quantum confinement effects in exfoliated black phosphorus. Nat. Mater. 14, 826-832 (2015).

52. Foo, E., Jaafar, M., Aziz, A. \& Sim, L. C. Properties of spin coated epoxy/silica thin film composites: effect of nano- and micron-size fillers. Composites, Part A 42, 1432-1437 (2011).

53. Mei, H., Landis, C. M. \& Huang, R. Concomitant wrinkling and buckle-delamination of elastic thin films on compliant substrates. Mech. Mater. 43, 627-642 (2011).

54. Madelung, O. Semiconductors: Data Handbook (Springer, Berlin, ed. 3, 2004).

55. Kim, H. et al. Synthetic $\mathrm{WSe}_{2}$ monolayers with high photoluminescence quantum yield. Sci. Adv. 5, eaau4728 (2019).

56. Gramling, H. M. et al. Spatially precise transfer of patterned monolayer $\mathrm{WS}_{2}$ and $\mathrm{MoS}_{2}$ with features larger than $10^{4} \mu \mathrm{m}^{2}$ directly from multilayer sources. ACS Appl. Electron. Mater. 1, 407-416 (2019). 
57. Nguyen, V. et al. Deterministic assembly of arrays of lithographically defined $\mathrm{WS}_{2}$ and $\mathrm{MoS}_{2}$ monolayer features directly from multilayer sources into van der Waals heterostructures. J. Micro Nano-Manuf. 7, 041006 (2019).

58. Huang, Y. et al. Degradation of black phosphorus (BP): the role of oxygen and water. Preprint at https://arxiv.org/abs/1511.09201 (2016).

59. Qiao, J., Kong, X., Hu, Z. X., Yang, F. \& Ji, W. High-mobility transport anisotropy and linear dichroism in few-layer black phosphorus. Nat. Commun. 5, 1-7 (2014).

60. Desai, S. B. et al. Strain-induced indirect to direct bandgap transition in multilayer $\mathrm{WSe}_{2}$. Nano Lett. 14, 4592-4597 (2014).

61. Matthewson, M. J., Kurkjian, C. R. \& Gulati, S. T. Strength measurement of optical fibers by bending. J. Am. Ceram. Soc. 69, 815-821 (1986).

62. Plechinger, G. et al. Control of biaxial strain in single-layer molybdenite using local thermal expansion of the substrate. 2D Mater. 2, 015006 (2015).

63. Luo, W., Song, Q., Zhou, G., Tuschel, D. \& Xia, G. Study of black phosphorus using angleresolved polarized Raman spectroscopy with $442 \mathrm{~nm}$ excitation. Preprint at https://arxiv.org/abs/1610.03382 (2016).

64. Zhang, Z. M., Lefever-Button, G. \& Powell, F. R. Infrared refractive index and extinction coefficient of polyimide films. Int. J. Thermophys. 19, 905-916 (1998).

65. Sherrott, M. C. et al. Anisotropic quantum well electro-optics in few-layer black phosphorus. Nano Lett. 19, 269-276 (2019). 
66. Beal, A. R. \& Hughes, H. P. Kramers-Kronig analysis of the reflectivity spectra of $2 \mathrm{H}-$ $\mathrm{MoS}_{2}, 2 \mathrm{H}-\mathrm{MoSe}_{2}$ and 2H-MoTe 2. J. Phys. C 12, 881 (1979).

67. Hori, Y., Ando, Y., Miyamoto, Y. \& Sugino, O. Effect of strain on band structure and electron transport in InAs. Solid State Electron. 43, 1813-1816 (1999).

68. Delimitis, A. et al. Strain distribution of thin InN epilayers grown on (0001) GaN templates by molecular beam epitaxy. Appl. Phys. Lett. 90, 061920 (2007).

69. Orsal, G. et al. Bandgap energy bowing parameter of strained and relaxed InGaN layers. Opt. Mater. Express 4, 1030-1041 (2014).

70. Varshni, Y. P. Band-to-band radiative recombination in groups IV, VI, and III-V semiconductors (I). Phys. Status Solidi 19, 459 (1967).

71. Vodopyanov, K. L., Graener, H., Phillips, C. C. \& Tate, T. J. Picosecond carrier dynamics and studies of Auger recombination processes in indium arsenide at room temperature. Phys. Rev. B. 46, 13194 (1992).

72. Rogalski, A. \& Jóźwikowski, K. The intrinsic carrier concentration in $\mathrm{Pb}_{1-\mathrm{x}} \mathrm{Sn}_{\mathrm{x}} \mathrm{Te}, \mathrm{Pb}_{1-}$ ${ }_{\mathrm{x}} \mathrm{Sn}_{\mathrm{x}} \mathrm{Se}$, and $\mathrm{PbS}_{1-\mathrm{x}} \mathrm{Se}_{\mathrm{x}}$. Phys. Status Solidi 111, 559 (1989).

73. Klann, R., Höfer, T. \& Buhleier, R. Fast recombination processes in lead chalcogenide semiconductors studied via transient optical nonlinearities. J. Appl. Phys. 77, 277 (1995).

74. Marchetti, S., Martinelli, M. \& Simili, R. The Auger recombination coefficient in InAs and GaSb derived from the infrared dynamical plasma reflectivity. J. Phys. Condens. Matter 14, 3653-3656 (2002). 


\section{Data availability}

All data generated or analysed during this study are included in this published article.

Acknowledgements The authors thank H. M. Fahad for help with gas sensing instrument setup.

Funding: Luminescence studies were supported by the U.S. Department of Energy, Office of Science, Office of Basic Energy Sciences, Materials Sciences and Engineering Division, under contract DE-AC02-05CH11231 within the Electronic Materials Program (KC1201). Photodetector fabrication and testing were supported by the Defense Advanced Research Projects Agency under contract no. HR0011-16-1-0004. K.B.C. acknowledges funding from the Australian Research Council (DP180104141 and FT140100577). H.K. acknowledges the support from a Samsung Scholarship.

Author contributions: H.K. and A.J. conceived the idea for the project and designed the experiments. H.K., S.Z.U., and D.-H.L. performed optical measurements. H.K., M.Y., and T.K. fabricated devices. S.Z.U., N.S.A., S.B., and K.B.C. performed optical simulations. N.G. helped with gas sensing experiments. Y.R. and C.P.G. helped with Raman measurements. H.K. wrote the manuscript. All authors discussed the results and commented on the manuscript.

\section{Competing interests:}

The authors declare no competing financial interests. 
a

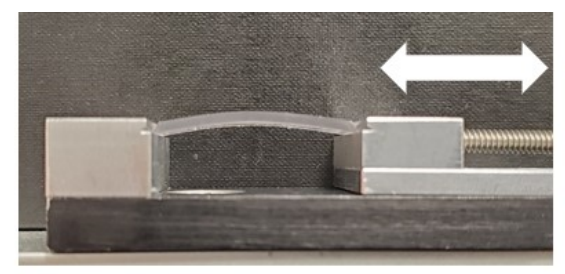

C $\quad$ Zero strain
$\quad$ Compressive strain
$\quad$ Tensile strain

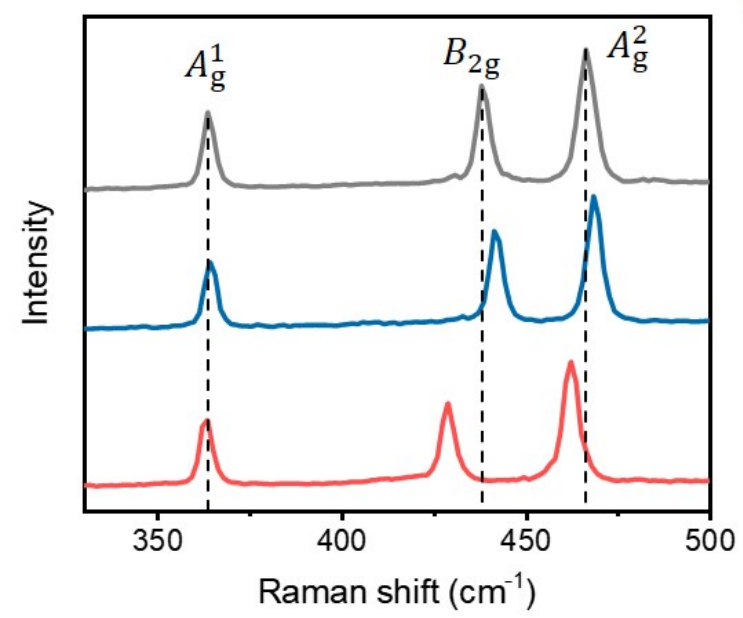

b

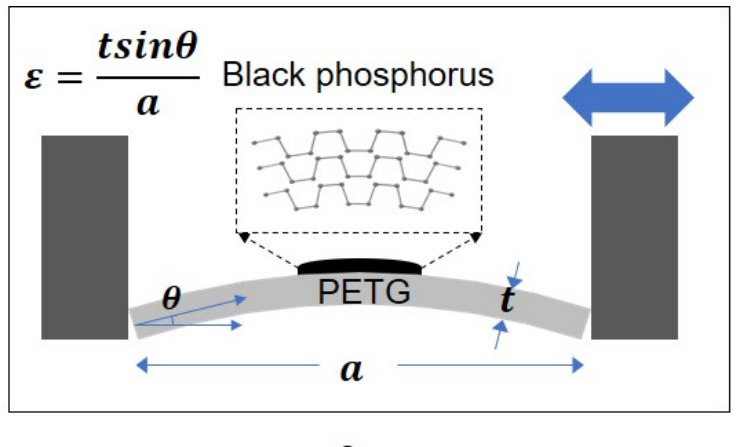

d
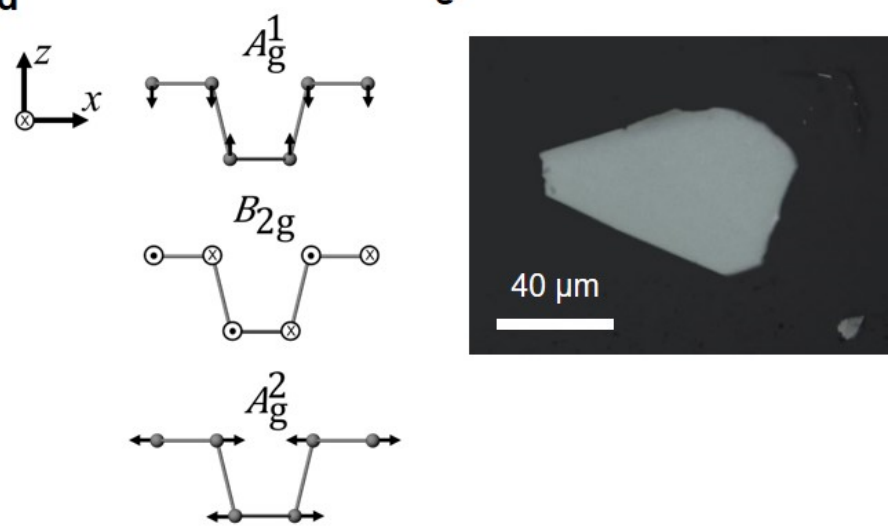

Extended Data Fig. 1 | Strain applied in bP. a, Photographic image of two-point bending apparatus setup used in this work. An electrical linear actuator that can push/pull the one point of the two-point bending apparatus applies the continuous and precise amount of uniaxial tensile strain in bP. b, Schematic of the two-point bending apparatus. The strain is calculated as $\varepsilon=t \sin \theta / a$ where $\varepsilon$ is the amount of strain, $t$ is the thickness of the substrate, $a$ is the length of the substrate, and $\theta$ is the angle representing the bending, which is equal to $a /(2 R)$ where $R$ is the radius of the curvature ${ }^{60}$. Note that the circular arc approximation is not satisfied when $\theta$ is large at the strain $>\sim 20 \%{ }^{61}$. c, Raman spectra of the bP measured in Fig. 1 and Fig. 2. d, Schematic of the atomic vibrations corresponding to Raman modes of $A_{\mathrm{g}}^{1}$ (out-of-plane), $B_{2 \mathrm{~g}}$ (in-plane, 
zigzag), and $A_{\mathrm{g}}^{2}$ (in-plane, armchair). e, Optical image of the strained bP flake on the PETG substrate. 
a

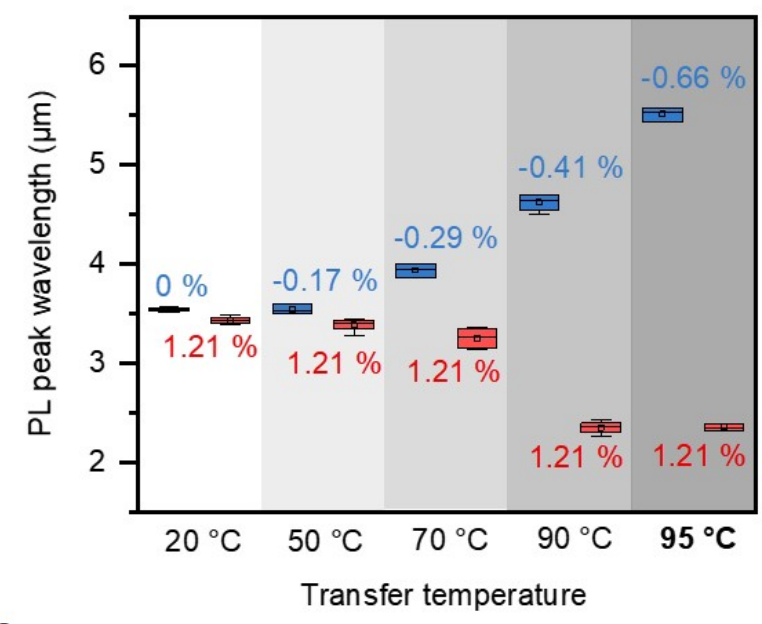

C

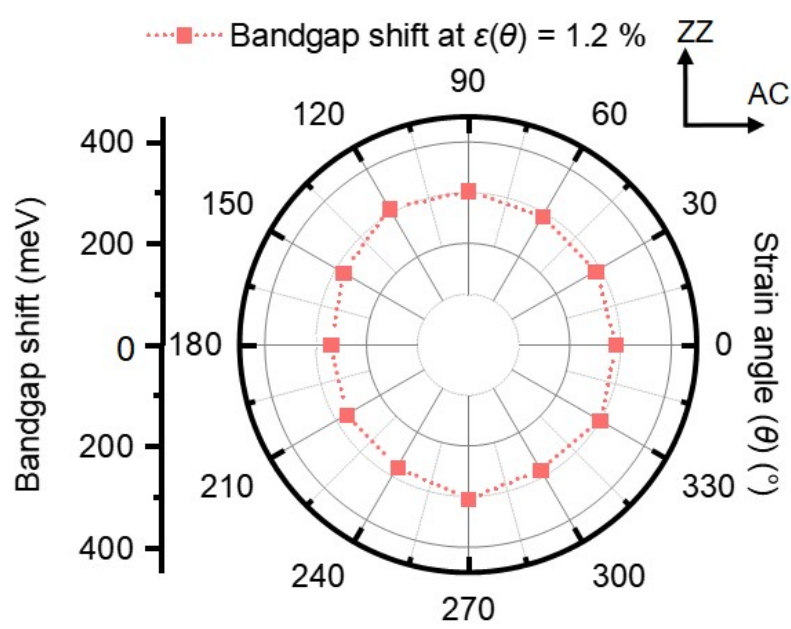

b

- Zero strain

- Compressive strain

- Tensile strain

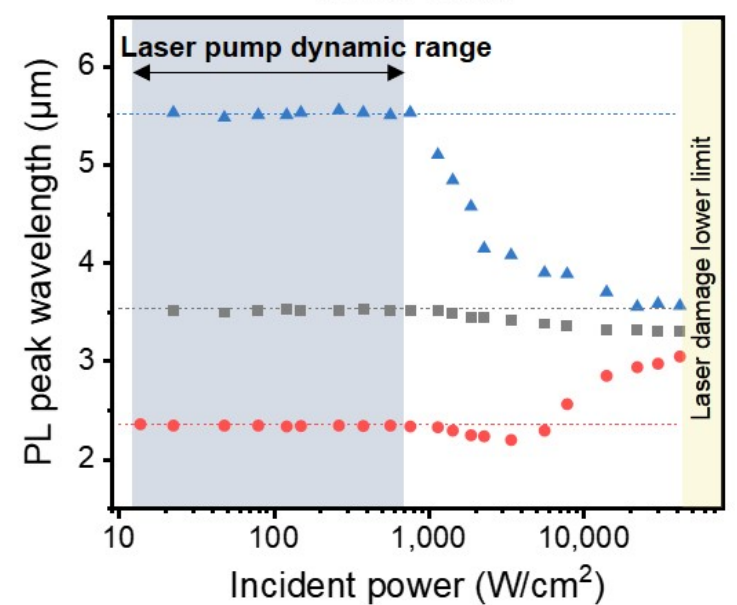

d

-Z Zero strain

- - Compressive strain

—- Tensile strain

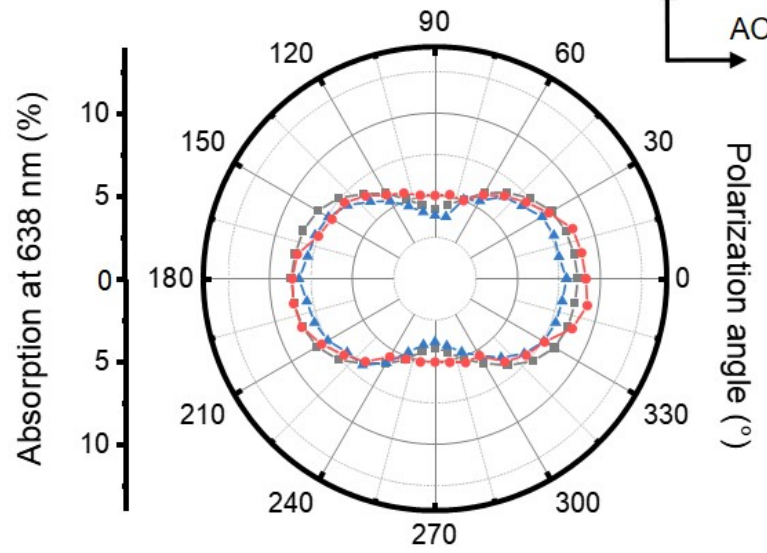

e

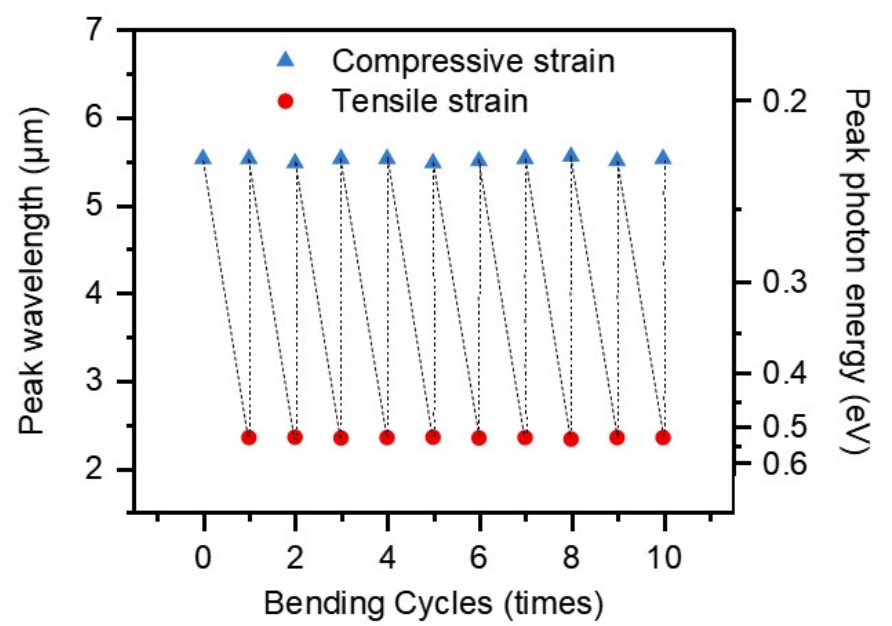


Extended Data Fig. 2 | Detailed optical characterization of strained bP. a, PL peak wavelength as a function of transfer temperature. The PL peak wavelength as a function of biaxial compressive strain before and after application of tensile strain $(1.21 \%, \mathrm{ZZ})$ was characterized. Each measurement was performed for five samples of bP with thickness 20-22 $\mathrm{nm}$. Samples were compressively strained by different amounts via different transfer temperature $\left(T_{\text {tr }}=20^{\circ} \mathrm{C}, 50^{\circ} \mathrm{C}, 70^{\circ} \mathrm{C}, 90^{\circ} \mathrm{C}\right.$, and $\left.95^{\circ} \mathrm{C}\right)$. It was observed that the PL peak shift resulting from tensile strain increased with increasing biaxial compressive strain (as determined by transfer temperature). This is understood to be the result of the following. At high biaxial strain, the larger friction-induced resistance prevents the sliding of the $2 \mathrm{D}$ materials ${ }^{55}$. At lower transfer temperatures, i.e. with reduced biaxial compressive strain, the bP is thus more likely to slip during bending of the substrate, making the intended uniaxial tensile strain not be efficiently delivered to the bP. This could be due to the fact that at lower values of biaxial strain (i.e. lower transfer temperatures), the friction-induced resistance that would prevent the sliding of the bP is reduced. b, Laser power dependence of strain effect. We characterized the laser-induced heating effect on the bandgap shift by measuring PL peak wavelengths as a function of laser power. Since the excitation spot size was about similar or slightly smaller than the size of the bP, it helped preventing the thermal expansion of the surrounding PETG by laser excitation, which could have acted as unexpected strain/slippage of bP from the PETG ${ }^{62}$. Regardless of the strain in $\mathrm{bP}$, the excitation power higher than $1500 \mathrm{~W} / \mathrm{cm}^{2}$ always resulted in the blueshift of the PL, which is attributed to the thermal heating by laser ${ }^{63}$. Although the PL peak position was recovered after cooling down to room temperature without the excitation, when the laser intensity was even higher $\left(>\sim 20 \mathrm{~kW} / \mathrm{cm}^{2}\right)$, there was a visible damage in bP, which did not 
return to its original PL peak position. Therefore, our laser incident power for the PL measurement in this work was maintained below $600 \mathrm{~W} / \mathrm{cm}^{2}$, at which power the PL peak of the exfoliated sample also remained constant without any shift. Also, this is the power range much lower than the lowest laser pump $\left(\sim 20 \mathrm{~kW} / \mathrm{cm}^{2}\right)$ which is known to have a laser thermal effect in bP and $\mathrm{MoS}_{2}$ transferred on polyimide or $\mathrm{PDMS}^{62,63}$. c, Bandgap shift under different strain directions with respect to the crystal orientation of bP. With changing direction of tensile strain with respect to the crystal orientation of bP, there was no apparent difference in the amount of strain-induced bandgap shift. This observation is consistent with previous results from a similar bending experiment performed on $6 \mathrm{~L}$ bP atop a polyethylene terephthalate (PET) substrate ${ }^{22}$. d, Absorption at excitation wavelength for bP without strain and bP with compressive strain and with $1.21 \%$ of uniaxial tensile strain. It was observed that the strain did not have significant effects on the light absorption of $\mathrm{bP}$ at the PL excitation wavelength. Even though the bP bandgap was being modulated by strain, because our excitation wavelength was far from the absorption edge the PL enhancement cannot be attributed to increased absorption. e, The reversibility and repeatability of the bandgap tuning in bP using compressive strain $(0.6 \%)$ and tensile strain (1.2\%) are demonstrated. The PL peak from $20 \mathrm{~nm}$ bP is shifted and recovered throughout the 10 cycles of bending and relaxation. At much higher strain, the PETG turns into plastic deformation, exhibiting no return to its original state. 
a

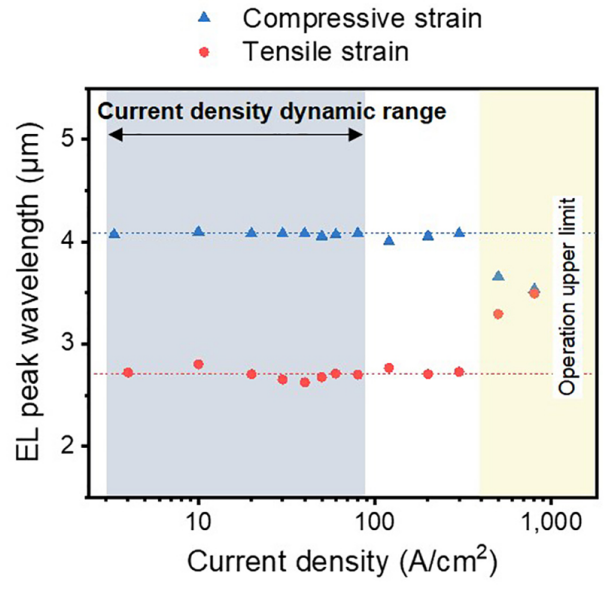

C

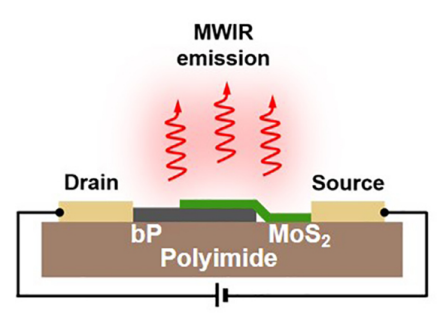

b

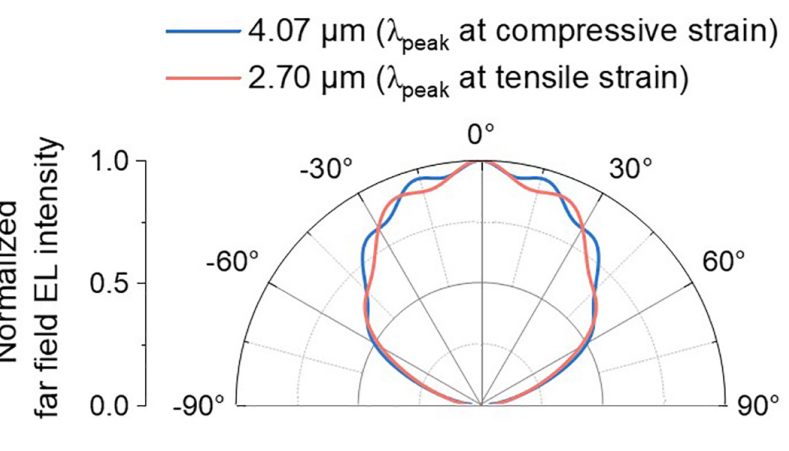

d

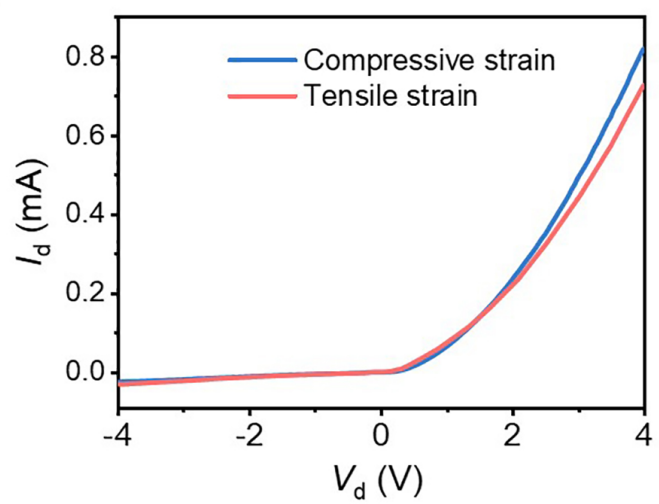

Extended Data Fig. 3 | Detailed characterization of variable spectrum bP-LED. a, Current density dependence of EL peak wavelength showing the reliability of strain-tunable emission in different injection levels. To prevent the degradation at elevated temperature and minimize the effect of the localized hot spots on the device performance, a polyimide film with high thermal conductivity was used in this study, coupled with Peltier module, to facilitate heat dissipation and keep the constant temperature during operation. Moreover, the forward current density was maintained below $20 \%$ of the lowest injection level where thermal failure started to take place. When the current density was high, the devices failed earlier and the visible degradation was observed in the channel region. Therefore, the current levels were maintained within the range shown in the figure $\left(4 \mathrm{~A} / \mathrm{cm}^{2} \sim 90 \mathrm{~A} / \mathrm{cm}^{2}\right)$ and the device showed stable operations over $\sim 8$ hours, 
which will be discussed later (Extended Data Fig. 5d,e). b. Angular intensity distribution of the strain-engineered bP-LED calculated with FDTD simulations (FDTD Solutions, Lumerical). c, Table showing the complex refractive indices of the polyimide substrate, bP, and $\mathrm{MoS}_{2}$ from the literature ${ }^{28,47,64-66}$, which was used for the simulation. While the precise computation awaits further study on the refractive index changes of bP with strains (compressive strain and tensile strain), we simply calculated the angular distribution of bP-LED at two different peak wavelengths, using the refractive indices of bP without strain from the literature. It was shown that the angular distributions at two different wavelengths were close enough to assume that there was no discrepancy between the power collections at these two emission wavelengths using the objective lens with a fixed collection angle. d, $I-V$ curves of strain-tunable bP/MoS 2 LED measured at compressive strain and $1.06 \%$ of tensile strain. 

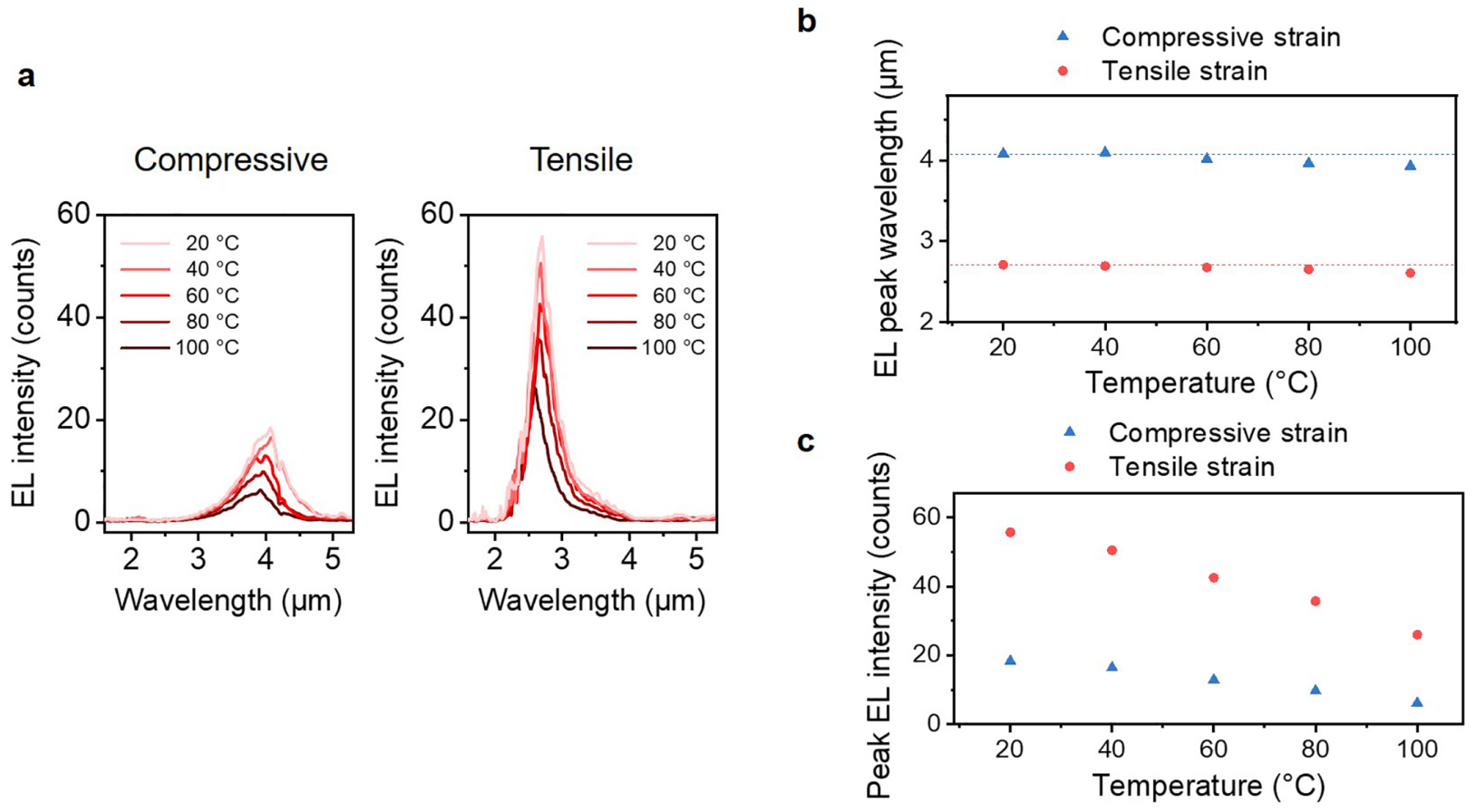

Extended Data Fig. 4 | Temperature-dependent performance of strain-tunable bP-LED. a, EL spectra for bP-LED on polyimide substrate operating at constant current density of $20 \mathrm{~A} / \mathrm{cm}^{2}$ at different temperatures under compressive strain $(0.2 \%)$ and tensile strain $(1.0 \%)$. b,c, Peak wavelength (b) and peak intensity (c) of the EL from bP-LED under different strains at different temperature. Note that, for heat dissipation and uniform temperature control during the device operation, dry nitrogen gas was consistently purged, and a mechanically flexible heat sink was installed, which was connected to the cold finger of the cryostat. 
b

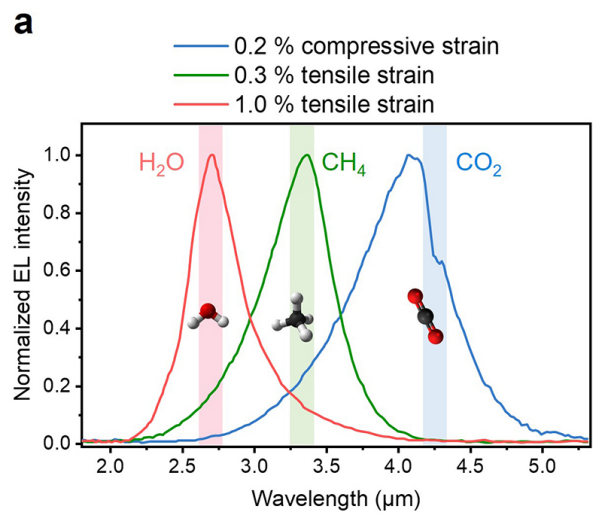

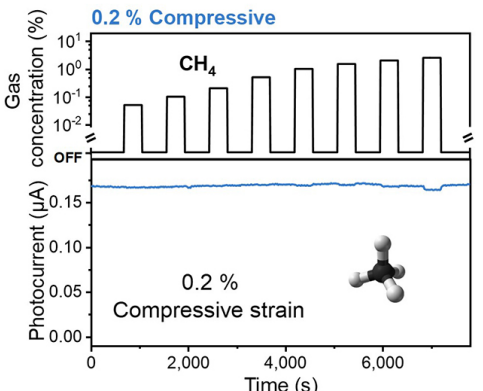

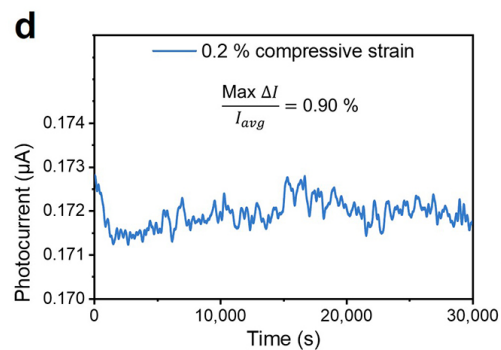

C
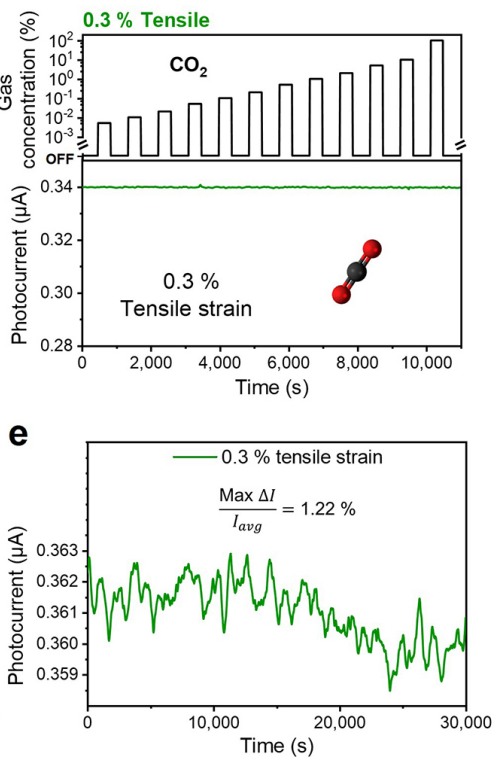

Extended Data Fig. 5 | Tuning emission wavelengths for detection of different gases. a,

Normalized EL spectra of bP-LED with compressive strain, $0.3 \%$ tensile strain, and $1.0 \%$ tensile strain for detecting $\mathrm{CO}_{2}, \mathrm{CH}_{4}$, and $\mathrm{H}_{2} \mathrm{O}$, respectively. b,c, The sensor response from the device under compressive strain in presence of $\mathrm{CH}_{4}$ gas $(\mathbf{b})$ and $0.3 \%$ of tensile strain in presence of $\mathrm{CO}_{2}$ gas (c). Our approach showed minimal detection at a concentration of $2.5 \%$ under compressive strain, while at $0.3 \%$ of tensile strain, it no longer showed the ability to detect $\mathrm{CO}_{2}$ gas. d,e, The stability of the gas-sensing setup for the bP-LED measured under compressive strain (d) and $0.3 \%$ of tensile strain (e). Both measurements were performed at the current density of $20 \mathrm{~A} / \mathrm{cm}^{2}$ with $f_{\text {mod }}=1 \mathrm{kHz}$. Over 8 hours of measurement, the device exhibited a maximum drift of $0.90 \%$ and $1.22 \%$ for compressive strain and $0.3 \%$ tensile strain, respectively. 
a

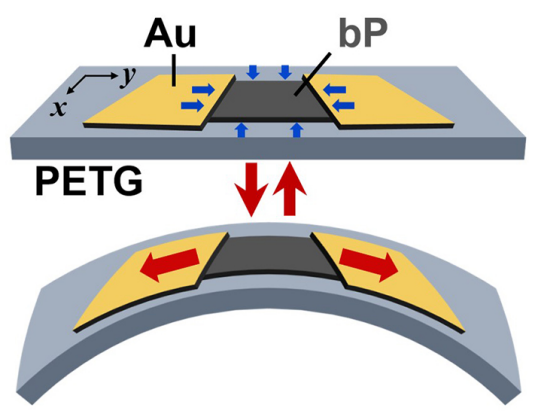

b

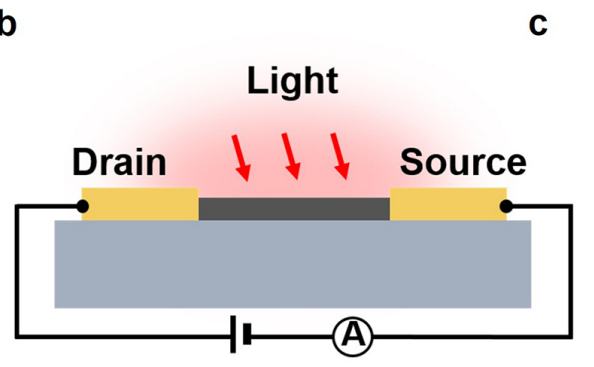

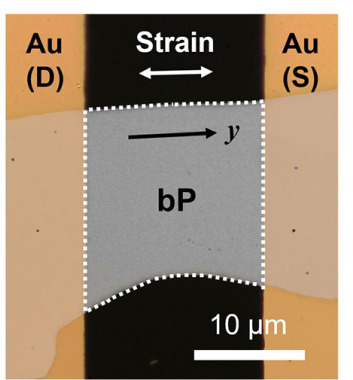

d

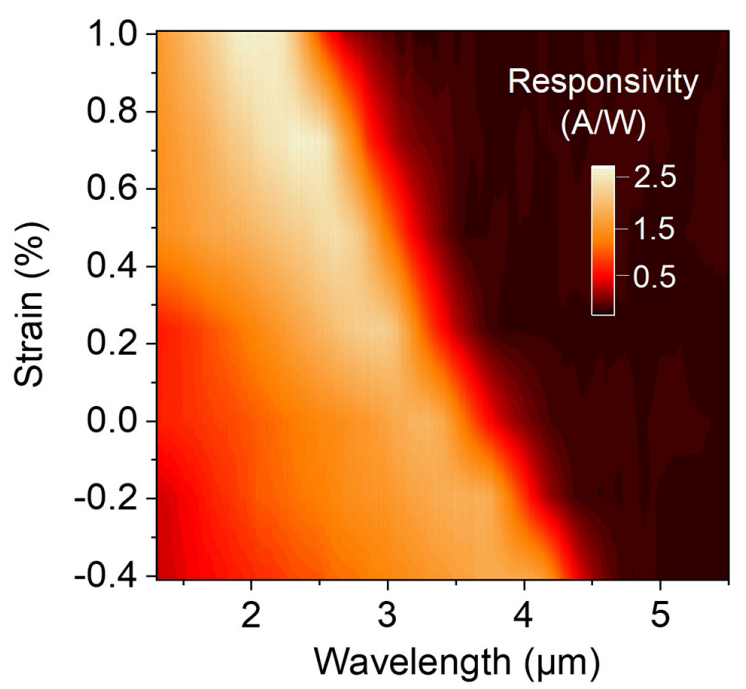

e

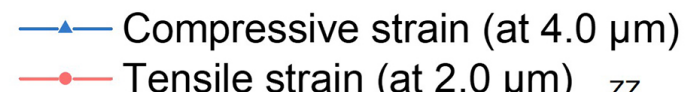

$\longrightarrow$ Tensile strain $($ at $2.0 \mu \mathrm{m}) \quad z z$
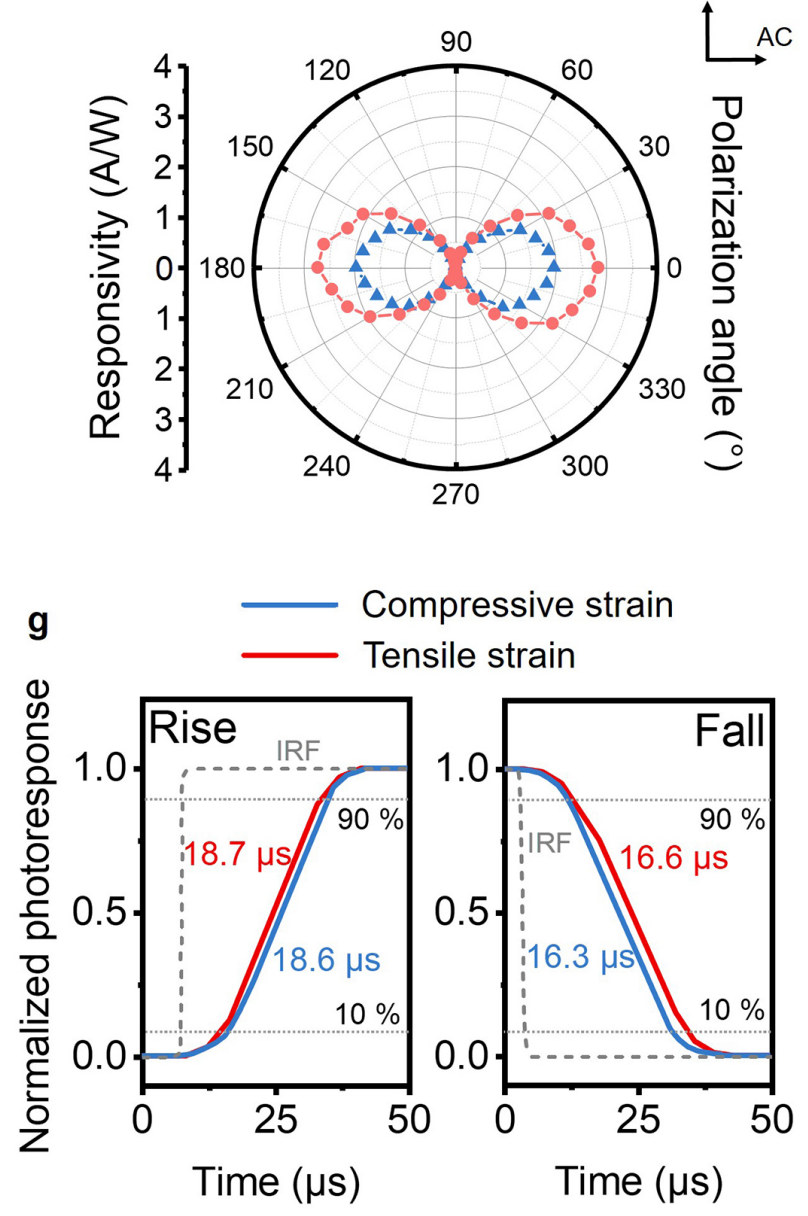

Extended Data Fig. 6 | Strain-tunable photoconductors based on bP. a, Schematic of a straintunable bP photoconductor. b, Schematic of device architecture, showing the photocurrent generation at a bias voltage. c, Optical micrograph of the device. d, Strain-dependent spectral 
photoresponse. e, Polarization-dependent responsivity at $4.0 \mu \mathrm{m}$ and $2.0 \mu \mathrm{m}$ for the device under compressive and $1.0 \%$ of tensile strain, respectively. f, $I-V$ curves of strain-tunable bP photoconductor measured in the dark and under illumination by a $1000 \mathrm{~K}$ black body. g, Rise and fall times (10-90\%) under $0.4 \%$ compressive and $1.0 \%$ tensile strain, using $1650 \mathrm{~nm}$ laser at $10 \mathrm{~mW} / \mathrm{cm}^{2}$. All measurements were conducted at a bias voltage of $100 \mathrm{mV}$ from a device with 22 nm bP thickness. 

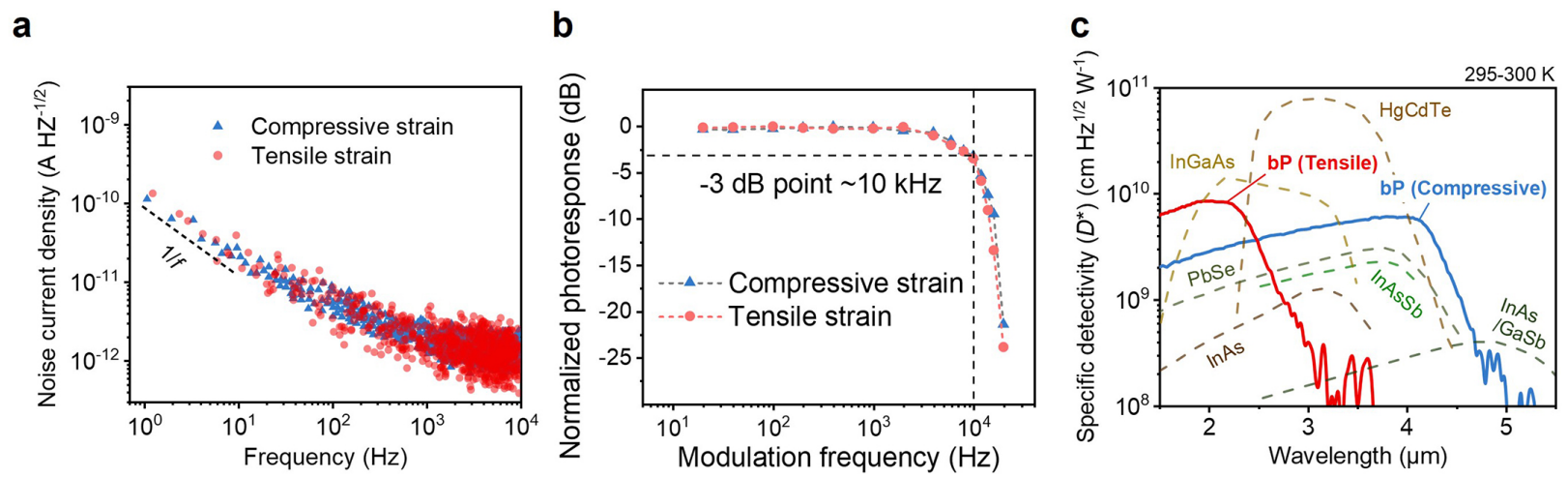

\section{Extended Data Fig. 7 | Detailed characterization of variable spectrum bP-photoconductor. a,}

Spectral noise density under $0.4 \%$ compressive and $1.0 \%$ tensile strain. Dashed line indicates the $1 / f$ curve at low frequency. $\mathbf{b}$, Normalized photoresponse of strain-tunable bP photoconductor measured as a function of modulation frequency. The device is measured at $V_{\mathrm{d}}=100 \mathrm{mV}$ and excited by a $1650 \mathrm{~nm}$ laser, showing a $3-\mathrm{dB}$ frequency of $10 \mathrm{kHz}$. c, Specific detectivity $\left(D^{*}\right)$ as a function of wavelength at room temperature, for the device with $0.4 \%$ compressive and $1.0 \%$ tensile strain as well as various commercially available photodetectors. 

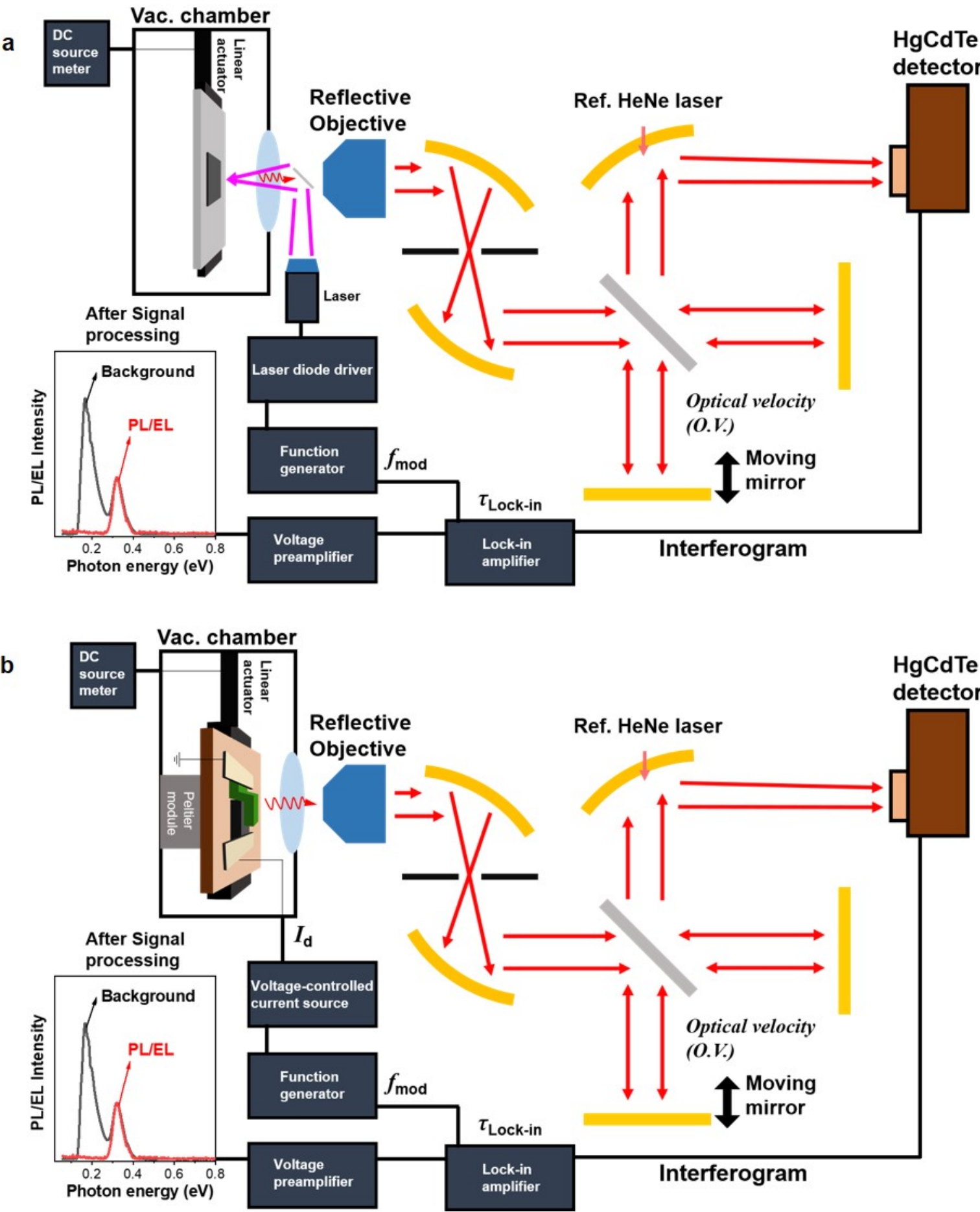

Extended Data Fig. 8 | PL and EL measurement setup. a, Schematic diagram of the IRPL

measurement setup and $\mathbf{b}$, Schematic diagram of the IREL measurement setup used in this work. 
For both measurements, the emission from bP was collected by a reflective objective and sent to the external port of the FTIR, with $f_{\text {mod }}=5 \mathrm{kHz}, \tau_{\text {Lock-in }}=300 \mu \mathrm{s}$, and $O . V .=0.0633 \mathrm{~cm} / \mathrm{s}$. Both the total interferogram from the $\mathrm{HgCdTe}$ (MCT) detector and the modulated interferogram from the current amplifier were utilized to separate the PL/EL signal from the thermal background. 


\begin{tabular}{cccccc}
\hline Material & $\begin{array}{c}\text { Bandgap shift } \\
\text { rate } \\
(\mathrm{meV} / \%)\end{array}$ & $\begin{array}{c}\text { Bandgap } \\
(\varepsilon=0 \%) \\
(\mathrm{eV})\end{array}$ & \multicolumn{2}{c}{ Applied strain } & Reference \\
\cline { 4 - 5 } $\mathrm{bP}$ & 162 & 0.35 & Uniaxial $(\mathrm{ZZ})$ & $-0.66 \sim 1.21$ & This work \\
$\ln \mathrm{As}$ & 36 & 0.35 & Biaxial & -3.2 & {$[67]$} \\
$\operatorname{lnN}$ & 10 & 0.7 & Biaxial & -9.9 & {$[68][69]$} \\
$\mathrm{Ge}$ & 62 & 0.67 & Biaxial & $-0.13 \sim 1.78$ & {$[26]$} \\
\hline
\end{tabular}

Extended Data Table 1 | Strain-induced bandgap shift of semiconductors in the SWIR/ MWIR range. 


\begin{tabular}{ccccc}
\hline Material & $n_{\mathrm{i}}\left(\mathrm{cm}^{-3}\right)$ & $B\left(\mathrm{~cm}^{3} / \mathrm{s}\right)$ & $C\left(\mathrm{~cm}^{6} / \mathrm{s}\right)$ & References \\
\hline InAs & $1.0 \times 10^{16}$ & $1.1 \times 10^{-10}$ & $1.1 \times 10^{-26}$ & {$[70][71]$} \\
$\mathrm{PbSe}$ & $3.0 \times 10^{16}$ & $8.0 \times 10^{-11}$ & $1.1 \times 10^{-28}$ & {$[72][73]$} \\
$\mathrm{GaSb}$ & $9.6 \times 10^{11}$ & $3.8 \times 10^{-10}$ & $9.3 \times 10^{-28}$ & {$[70][74]$} \\
$\mathrm{bP}(25 \mathrm{~nm})$ & $2.5 \times 10^{15}$ & $2.7 \times 10^{-21}$ & $4.1 \times 10^{-43}$ & {$[29]$} \\
\hline
\end{tabular}

Extended Data Table 2 | Parameters used to calculate theoretical QY from ABC model. 


\section{University Library}

\section{- M M N E R VA A gateway to Melbourne's research publications}

Minerva Access is the Institutional Repository of The University of Melbourne

\section{Author/s:}

Kim, H;Uddin, SZ;Lien, D-H;Yeh, M;Azar, NS;Balendhran, S;Kim, T;Gupta, N;Rho,

Y;Grigoropoulos, CP;Crozier, KB;Javey, A

Title:

Actively variable-spectrum optoelectronics with black phosphorus

\section{Date:}

2021-08-12

\section{Citation:}

Kim, H., Uddin, S. Z., Lien, D. -H., Yeh, M., Azar, N. S., Balendhran, S., Kim, T., Gupta, N., Rho, Y., Grigoropoulos, C. P., Crozier, K. B. \& Javey, A. (2021). Actively variablespectrum optoelectronics with black phosphorus. NATURE, 596 (7871), pp.232-+. https:// doi.org/10.1038/s41586-021-03701-1.

Persistent Link:

http://hdl.handle.net/11343/294844 